\title{
The LCN2/LOXL2/MMP9 ternary protein complex promotes migration and invasion through the FAK/AKT/GSK3 $\beta$ signaling pathway in esophageal squamous cell carcinoma
}

\section{Qiaoxi Xia}

Shantou University Medical College

Mantong Chen

Shantou University Medical College

Xiao Zhou

Shantou Central Hospital

Wenjing Bai

Shantou University Medical College

Ling Lin

Shantou University Medical College

Yan Zhao

Shantou Central Hospital

Jiyu Ding

Shantou University Medical College

Xiaoqi Zheng

Shantou University Medical College

Haiying Zou

Shantou University Medical College

Shaohong Wang

Shantou Central Hospital

Liyan Xu

Shantou University Medical College

Enmin Li

Shantou University Medical College

Zepeng Du

Shantou Central Hospital

Bingli Wu ( $\nabla$ blwu@stu.edu.cn )

Shantou University Medical College https://orcid.org/0000-0002-0614-4721 
Research

Keywords: Extracellular matrix, Protein ternary complex, Migration, Invasion

Posted Date: October 5th, 2021

DOI: https://doi.org/10.21203/rs.3.rs-919307/v1

License: (c) (i) This work is licensed under a Creative Commons Attribution 4.0 International License. Read Full License 


\section{Abstract \\ Background}

During tumor malignant development, the extracellular matrix (ECM) is usually abnormally regulated. Dysregulated expression of lysyl oxidase-like 2 (LOXL2), matrix metalloproteinase 9 (MMP9) and lipocalin 2 (LCN2) are associated with ECM remodeling. Nevertheless, the evidence of an LCN2/LOXL2/MMP9 protein complex and its potential molecular mechanisms remain unclear.

\section{Methods}

In this study, the LCN2/LOXL2/MMP9 ternary complex and their specific interaction domains were determined by co-immunoprecipitation. Next, biological functions of the complex were assessed by wound-healing, migration, invasion and MTS assays. Xenografts were applied to study the effect of the LCN2/LOXL2/MMP9 ternary complex on the tumor progression in vivo. Finally, the molecular mechanism of this ternary complex was explored by zymography, immunofluorescence, and fluorescent matrix degradation.

\section{Results}

Protein-protein interaction assays indicated LCN2 and LOXL2, and LCN2 and MMP9 both had intracellular and extracellular interactions, but the interaction between LOXL2 and MMP9 only occurred intracellularly. The LCN2/LOXL2/MMP9 ternary complex promoted migration and invasion of ESCC cells, as well as tumor growth and malignant progression in vivo, while the iron chelator deferoxamine mesylate (DFOM) inhibited ESCC tumor growth. Co-overexpression of LCN2/LOXL2/MMP9 enhanced the ability of tumor cells to degrade fibronectin and Matrigel, increased the formation and extension of filopodia, promoted the rearrangement of microfilaments through upregulation of profilin 1. In addition, the LCN2/LOXL2/MMP9 ternary complex promoted the expression of SPOCK1, and abnormally activated the FAK/AKT/GSK3 $\beta$ signaling pathway.

\section{Conclusion}

The LCN2/LOXL2/MMP9 ternary complex promotes the migration and invasion of cancer cells and malignant tumor progression through multiple mechanisms, which could be a potential therapeutic target.

\section{Background}

Esophageal cancer currently ranks seventh in terms of incidence $(604,000$ new cases) and sixth in mortality overall $(544,000$ deaths), the latter signifying that esophageal cancer was responsible for one in 
every 18 cancer deaths in 2020 [1]. The global incidence of esophageal cancer has increased significantly in the past four decades [2], with esophageal squamous cell carcinoma (ESCC) being the most common histologic subtype worldwide, and China being one of the countries with a high incidence of ESCC [3]. Esophageal carcinoma cannot be easily diagnosed at the early stages, leading to most patients being diagnosed at an advanced stage, thus losing the opportunity for early treatment. Compounding the problem, the malignant pathogenesis of esophageal carcinoma is still not fully understood.

Tumor progression is strongly linked with the tumor microenvironment. The extracellular matrix (ECM) is the main component of microenvironment, and production, secretion, degradation and rearrangement of the ECM are strictly regulated [4]. However, homeostasis of the ECM is dynamically disrupted during cancer progression. Matrix metalloproteinases (MMPs), especially MMP9, are thought to be particularly important for cancer invasion and metastasis, as a result of their ability to degrade ECM barriers. MMP9 is involved in multiple biological processes, such as proteolytic degradation of ECM, alteration of cell-cell and cell-ECM interactions, cleavage of cell surface proteins and cleavage of proteins in the extracellular environment [5].

LCN2, also known as neutrophil gelatinase-associated lipocalin (NGAL), is a secreted glycoprotein whose abnormal expression in various human solid tumors plays a vital role in epithelial-mesenchymal transformation (EMT), angiogenesis and cell migration and invasion [6-7]. LCN2 and MMP9 form a protein complex, to protect MMP9 from protein degradation, thereby prolonging MMP9 enzymatic activity [8]. The interaction of LCN2 with MMP-9 plays a crucial role in the modulation of the metastatic phenotype of cancer cells, and correlates with the aggressive behavior of neoplastic cells in several types of cancer [9]. In our previous study, we found LCN2 to be highly expressed and associated with poor prognosis for patients with ESCC [10]. Later, we also showed that LCN2 promotes the migration and invasion of ESCC cells to increase MMP-9 activity through a novel positive feedback loop [11].

On the other hand, LOXL2 (lysyl oxidase-like 2), a member of lysyl oxidase family, is a secreted copperdependent amine oxidase. One important role of LOXL2 is to catalyze the covalent cross-linking of collagen and elastin in the extracellular matrix (ECM), which contributes to strength of collagen fibrils and elasticity of elastic fibers [12]. We have found that high expression of LOXL2 is associated with lymph node metastasis and poor prognosis in ESCC, and that LOXL2 induces cytoskeletal reorganization and ezrin phosphorylation, which subsequently promotes tumor cell invasion and metastasis in ESCC [1314]. Accumulating evidence suggests LOXL2 can promote invasion and metastasis, angiogenesis and malignant transformation in many solid tumors, and aberrant high expression of LOXL2 usually is correlated with poor prognosis [15].

Taken together, LCN2, LOXL2 and MMP9 have emerged as promising therapeutic targets in many types of cancer $[9,15-16]$. Besides their individual diverse functions, LCN2, MMP9 and LOXL2 are all secreted proteins and share a common involvement in reshaping the ECM, contributing to the progression, invasion and migration of tumors. Therefore, we investigated whether LCN2, LOXL2 and MMP9 are 
interrelated in ECM remodeling, and whether LCN2/LOXL2/MMP9 form a complex to play a synergistic role in tumor progression.

\section{Materials And Methods}

\section{Cells and cell culture}

ESCC cell lines KYSE140, KYSE150, KYSE180, KYSE30, KYSE410, KYSE450, KYSE510 and TE3 were used for this study. The sources and STR genotyping of these cell lines have been described previously [11, 14]. ESCC cells and human embryonic kidney 293T cells were grown in RPMI-1640 (HyClone, USA) supplemented with 10\% heat-inactivated fetal bovine serum (Life Technology) and $1 \%$ penicillinstreptomycin (Meijin, China). Cells were cultured in a $5 \% \mathrm{CO}_{2}, 37^{\circ} \mathrm{C}$ cell culture incubator, and passaged by $0.25 \%$ trypsin digestion.

\section{Plasmid preparation}

Expression plasmids, including pcDNA3.1-LCN2, pcDNA3.1-LCN2-HA, pcDNA3.1-MMP9-Flag, pcDNA3.1LOXL2-HA, pcDNA3.1-LOXL2-Flag, DLOXL2(545-774aa)-HA, DLOXL2(1-544aa)-HA, were described in our previous studies [11, 17]. pCMV3-MMP9-Flag was purchased from Sino Biological (Beijing, China). Plasmids were maintained in Trans5a E. coli (CD201, TransGen, China), and were harvested by EZgene ${ }^{\mathrm{TM}}$ EndoFree Plasmid Miniprep Kit (PD1212-01, BIOMIGA, China). Plasmids were transfected with Lipofectamine 3000 (L3000-015, Thermo) according to the manufacturer's instructions. A cell line stably overexpressing LCN2/LOXL2/MMP9 was obtained by transfection using a Neon ${ }^{\mathrm{TM}}$ Transfection System (MPK5000S, Thermo), with the parameters of $1400 \mathrm{~V}, 20$-ms pulse width, 2 pulse), followed by selection in hygromycin B (10687010, Thermo) and Geneticin ${ }^{\text {TM }}$ Selective Antibiotic (G418 Sulfate, 10131027, Thermo) for 2 weeks.

\section{Protein extraction and concentration of conditioned medium}

Cells were seeded in a $10 \mathrm{~cm}$ dish (for co-IP) or $6 \mathrm{~cm}$ dish (for western blotting), cultured for $36 \mathrm{~h}$ following transfection, and then starved for $12 \mathrm{~h}$ in serum-free medium. Cells were then lysed in RIPA buffer $(9806 \mathrm{~S}, \mathrm{CST})$ to extract the total protein. Conditioned medium was concentrated using Amicon ${ }^{\circledR}$ Ultra centrifugal filters (UFC5010BK, MILLPORE) by centrifugation at $14,000 \times \mathrm{g}$ for $10-30 \mathrm{~min}$, to a final volume of approximately $20 \mu \mathrm{l}$ for each sample.

\section{Western blotting}

Approximately $30 \mu \mathrm{g}$ of total protein was loaded, separated by $10 \%$ SDS-PAGE, and transferred to PVDF membranes (3010040001, Roche BR). The membranes were blocked with 5\% skim milk powder (P0101, Maygene) in TBST (E175-01, GenStar) for $1 \mathrm{~h}$ and incubated with primary antibodies, then incubated with an HRP-conjugated secondary antibody. The specific primary antibodies and secondary antibodies used in this study are provided in Supplementary Table 1. Finally, chemiluminescence was performed using 
SuperSignal ${ }^{\mathrm{TM}}$ West Pico PLUS Chemiluminescent Substrate (34579, Thermo) to visualize the protein bands of interest by a Bio-Rad ChemiDoc Imaging System (Bio-Rad, Hercules, CA, USA).

\section{Co-immunoprecipitation}

The cells were seeded into a $10 \mathrm{~cm}$ dish and transfected with the following pairs of plasmids: LCN2HA/LOXL2-Flag, LCN2-HA/MMP9-Flag, LOXL2-HA/MMP9-Flag, LCN2-HA/LOXL2-HA/MMP9-Flag, LCN2 and DLOXL2(1-544aa)-HA, LCN2 and DLOXL2(545-774aa)-HA, MMP9-Flag and DLOXL2(1-544aa)-HAX MMP9-Flag and DLOXL2(545-774aa)-HA, respectively. These transfected cells were cultured for $36 \mathrm{~h}$, then starved for $12 \mathrm{~h}$ in serum-free medium. Then total protein was extracted, and the conditioned medium was concentrated. These samples were subjected to co-immunoprecipitation using antiDYKDDDDK magnetic agarose (A36797, Thermo) or anti-HA magnetic beads (88836, Thermo), respectively, and then placed in a rotary mixer for $30 \mathrm{~min}$ at room temperature. The beads were washed three times with $1 \times$ PBS, boiled and eluted to obtain the conjugated proteins, which were subjected to anti-Flag or anti-HA immunoblotting analysis.

\section{Mass spectrometry analysis}

LCN2-HA and LOXL2-Flag plasmids were co-transfected as an experimental group, and LOXL2-Flag plasmid was transfected as a control group in KYSE150 cells. The whole cell lysate was coimmunoprecipitated with anti-Flag antibody, separated by SDS-PAGE and stained with Coomassie Brilliant Blue. A differentially-expressed protein band around 25kD was excised and digested with trypsin for mass spectrometry ( $Q$ Exactive, Thermo) analysis. The peptides were identified by searching against the Uniprot database (https://www.uniprot.org/).

\section{Wound healing assay}

A scratch wound assay was carried out to measure the distance of cell migration. After 24 hours of transfection, cells were trypsinized, suspended and adjusted to a cell density of $2.5 \times 10^{5} \mathrm{cell} / \mathrm{s} / \mathrm{ml}$, inoculated into a 12-well plate, cultured for 12 hours, and then starved for 12 hours in serum-free medium. A straight-line scratch was made on the monolayer culture, approximately $80-90 \%$ confluence, using a sterile $200 \mu \mathrm{L}$ pipette tip. The wells were photographed under 200X magnification every $6 \mathrm{~h}$ or $12 \mathrm{~h}$ with microscope (Olympus, Tokyo, Japan).

\section{Transwell migration and invasion assays}

Migration and invasion assays to measure the mobility of cancer cells were performed using transwell chambers as previously described [11]. Cells were cultured 24 hours after transfection, and then starved for 12 hours in serum-free medium. The cells were digested and adjusted to $1.25 \times 10^{5} \mathrm{cells} / \mathrm{ml}$ (migration assay), $2.5 \times 10^{5} \mathrm{cells} / \mathrm{ml}$ (invasion assay), then $400 \mu \mathrm{l}$ cell suspension was added to the upper chamber: uncoated transwells (353097, FALCON) were used for the migration assay, and Matrigel-coated transwells (356234, CORNING) were used for the invasion assay. Afterwards, $500 \mu \mathrm{l}$ of $10 \%$ serum medium was 
added to the lower chamber. Cells were incubated for $24-36 \mathrm{~h}$ (migration assay) or 36-48 h (invasion assay). At the end of the culture, non-migrated cells in the upper chamber were carefully removed with a cotton swab. The migrated cells that passed through the membrane and adhered to the lower surface of the membrane were fixed with $4 \%$ paraformaldehyde for $15 \mathrm{~min}$, and then stained with crystal violet for 5 min. The number of migrated/invaded cells in 10 random fields was photographed using a 200x microscope (IX73, OLYMPUS, Japan).

\section{MTS assay}

Transfected cells were trypsinized, suspended and adjusted to $1 \times 10^{5}$ cells $/ \mathrm{ml}$ with serum-containing medium. Then, $100 \mu \mathrm{l}$ of the cell suspension was added to each well of a 96 -well plate $(701001$, NEST) in triplicate. At 0, 24, 48, 72 and $96 \mathrm{~h}$ after attachment, $20 \mu$ of MTS reagent (G358B, Promega) was added to each well, and the OD of each well was measured using a Multiskan FC microplate reader (Thermo) after a $2 \mathrm{~h}$ incubation at $37^{\circ} \mathrm{C}$.

\section{Flow cytometry}

For flow cytometry analysis, Cells were seeded into 6-well plates and co-transfected with desired plasmids. After $36 \mathrm{~h}$ of growth, the cells were trypsinized and centrifuged (700 rpm, $10 \mathrm{~min}$ ), and the conditioned medium was removed. The cells were resuspended in $600 \mu \mathrm{l}$ of $1 \times$ PBS and $1.4 \mathrm{ml}$ of precooled $70 \%$ ethanol, then incubated at $4^{\circ} \mathrm{C}$ overnight. The next day, the cells were pelleted by centrifugation and resuspended in $500 \mu \mathrm{lBS}, 2 \mu \mathrm{l}$ of propidium iodide (PI) (P4864-10ML, Sigma) and $12.5 \mu \mathrm{l}$ of RNase $(2 \mathrm{mg} / \mathrm{ml})$, followed by incubation in the dark for $30 \mathrm{~min}$ at room temperature. Samples were analyzed on a BD Accuri C6 flow cytometer (BD, Bioscience, Franklin Lakes, NJ, USA) and data analysis was performed using FlowJo software (Tree Star, Ashland, OR, USA).

\section{Colony-forming assay}

At 36 hours after transfection, 1,000-2,000 cells were seeded into 6-well plates and cultured for 2 weeks. When macroscopic clones were formed, cells were fixed in $4 \%$ paraformaldehyde for 20 min, stained with hematoxylin for $5 \mathrm{~min}$ and photographed with a ChemiDoc Imaging System. The number of clones was scored.

\section{Zymography analysis}

MMP9 activity was measured by gelatin zymography as previously described [11]. The conditioned medium of transfected cells was concentrated and subjected to SDS-PAGE electrophoresis with $1 \%$ gelatin. Following electrophoresis, the separation gel was washed twice in washing buffer $(2.5 \%$ Triton X100) for 30 minutes each wash, then incubated in incubation buffer $(40 \mathrm{mM}$ Tris- $\mathrm{HCl}(\mathrm{pH} 8.0), 10 \mathrm{mM}$ $\mathrm{CaCl}_{2}$ ) at $37^{\circ} \mathrm{C}$ for 24 hours. The gel was stained with $0.1 \%$ Coomassie blue dye for $1 \mathrm{~h}$, destained and fixed. The proteolytic activities of MMP9 and its complex were detected as a clear white zone against a Coomassie Blue-stained gel background. 


\section{Fluorescent matrix degradation assay}

Glass coverslips were coated with $0.01 \%$ poly-L-lysine (P8920-100ML, Sigma), air-dried overnight, and fixed with $0.5 \%$ glutaraldehyde. Fibronectin HiLyte 488 (FNR02-A, Cytoskeleton) was added dropwise to each glass coverslip, which was then transferred to a 24 -well plate and incubated at $37^{\circ} \mathrm{C}$ for $1 \mathrm{~h}$. Cells were suspended following trypsinization and added to the glass coverslips. After $48 \mathrm{~h}$, each glass coverslip was fixed, and cells were permeabilized and labeled with Acti-Stain ${ }^{\mathrm{TM}} 555$ Fluorescent phalloidin (PHDH1, Cytoskeleton) followed by mounting in anti-fluorescence attenuating reagent (S2110, Solarbio). For determination of matrix degradation, the coverslips were photographed using a laser confocal microscope (ZEISS LSM 800, Carl Zeiss, Jena, Germany).

\section{D cell in vitro culture}

Matrigel $^{T M}$ (BD Biosciences, Bedford, MA, USA) was thawed on ice, and diluted 1:1 with serum free-cold cell culture media. The transfected cells were trypsinized and adjusted to $1 \times 10^{6} \mathrm{cells} / \mathrm{ml}$, then $50 \mu \mathrm{l}$ diluted Matrigel and $10 \mu \mathrm{l}$ cell suspension were mixed in a 6-well plate, and incubated at $37^{\circ} \mathrm{C}$ for $30 \mathrm{~min}$. After solidifying, $3 \mathrm{ml}$ of $10 \%$ fresh serum medium was added, and cells were photographed under a $200 \times$ inverted microscope every 3-6 hours.

\section{Immunofluorescence}

Cells were seeded into 6-well plates and co-transfected with the desired plasmids. After $36 \mathrm{~h}$, the cells were trypsinized and inoculated into a 24-well plate containing coverslips and cultured for an additional $12-24 \mathrm{~h}$. The cells were fixed with $4 \%$ paraformaldehyde, permeabilized with $0.1 \%$ Triton X-100 (T0694$100 \mathrm{ML}$, AMRESCO) for $3 \mathrm{~min}$ at room temperature, then blocked with $5 \%$ donkey serum at room temperature for $1 \mathrm{~h}$. Primary antibodies were added and incubated at $4^{\circ} \mathrm{C}$ overnight, including antibodies against LCN2, LOXL2, MMP9, HA, Flag and profilin 1, respectively. Fluorescent secondary antibody was dropped onto the coverslips and incubated at room temperature for $1 \mathrm{~h}$ in the dark. The fluorescent secondary antibodies used herein included: Acti-Stain ${ }^{\mathrm{TM}} 555$ fluorescent phalloidin ( $\mathrm{PHDH} 1$, Cytoskeleton), Acti-Stain ${ }^{\text {TM }} 488$ fluorescent phalloidin (PHDG1, Cytoskeleton), Alexa Fluor 488 DAM ReadyProbes Reagent with NZ-derived BSA (R37114, Invitrogen), or Alexa Fluor 594 DAR ReadyProbes Reagent with NZ-derived BSA (R37119, Invitrogen). The slides were photographed with a laser confocal microscope (ZEISS LSM800).

\section{Xenograft assay in nude mice}

Animal experiments was conducted in accordance with protocols approved by the Animal Ethics Committee of Shantou University Medical College. Four-week-old nu/nu nude mice (Beijing Vital River Laboratory Animal Technology Co., Ltd, China) were randomized into five groups (6 mice/group), and their footpads or subcutaneous layer were inoculated with $1 \times 10^{7}$ cells of each stably transfected KYSE150 cell line or wildtype KYSE150 cells. Mouse general behavior was monitored, and tumor size was measured every 3 days for a total 30 days. For drug treatment experiments, nude mice injected 
subcutaneously were observed for the first 3 days, and then intraperitoneal injections of $30 \mu \mathrm{M}$ deferoxamine mesylate (DFOM) (HY-B0988, MCE) were given daily, for a total of 14 days. At the end of the experiment, the nude mice were euthanized and xenograft tumors, including footpad primary tumors and popliteal-infiltrated tumors, and popliteal lymph nodes were dissected, weighed, and analyzed as described previously [11]. The tumor sizes and lymph node volumes were calculated by the formula of width $\times$ length $\times$ height $/ 2$.

\section{Statistical analysis}

Data analyses were performed with SPSS 13.0 (SPSS, Inc., USA). Two-group comparisons were performed using Student's $t$-test. Differences between groups were assessed by one-way analysis of variance when more than two groups were compared. Differences were considered statistically significant at $* P<0.05$ and $* * P<0.01$. Pearson correlation analysis was used for the correlation test of the two groups of data. Experimental data are presented as the means \pm SD for at least three independent experiments.

\section{Results}

\section{Protein-protein interaction network and cell model construction}

To identify the proteins that interact with LOXL2, we performed co-IP using LOXL2 as bait followed by LCMS analysis. Mass spectrometry results showed that multiple LCN2 protein peptides were identified from the differentially-expressed 25kD band in LOXL2-overexpressing cells. The PEP values of the experimental group peptides were far less than 0.1 , and the cumulative coverage was as high as $42.3 \%$, indicating the results were highly credible (Supplementary Table 2). The secondary ion mass spectrum of the VPLQQNFQDNQFQGK proteolysis product of LCN2 protein is shown in Supplementary Fig. S1A. The peaks represented the secondary ion peaks of the peptide measured by the mass spectrum. These results indicated that LOXL2 and LCN2 interacted to form a complex.

Next, to gain a complete view of the interactome between LCN2 and LOXL2, all literature reporting interacting proteins was obtained from HPRD (http://www.hprd.org/) and BioGRID (http://thebiogrid.org/), and were processed and merged into a "parent network" as previously described [18]. The parental network was entered into the Cytoscape program to construct a secondary protein interaction sub-network using LCN2 and LOXL2 as the seed proteins. LCN2 was linked with LOXL2 by at least 5 pair partner proteins, further supporting that LCN2 interacts directly or physically with LOXL2 (Supplementary Fig. S1B). To understand their correlation in ESCC clinical samples, the Pearson correlations for LCN2/MMP9, LCN2/LOXL2 and MMP9/LOXL2 in the public GSE53625 dataset were examined. A significant correlation was found for LCN2/MMP9 and MMP9/LOXL2 (Supplementary Fig. S1C).

The endogenous expression level of LCN2 and LOXL2 was examined in eight esophageal cancer cell lines, with human 293 T as a control. KYSE410 and KYSE150 cells showed relatively low endogenous 
LCN2 and LOXL2 levels, and were selected as the cell model for subsequent experiments (Supplementary Fig. S1D). KYSE410 and KYSE150 cells were co-transfected with LCN2-HA/LOXL2-Flag, LCN2-HA/MMP9Flag, or LOXL2-HA/MMP9-Flag plasmid pairs, or the corresponding vector plasmids. High expression of the target proteins was observed in KYSE410 and KYSE150 after transfection, accordingly (Supplementary Fig. S1E).

\section{The interaction patterns of intracellular and extracellular LCN2/LOXL2/MMP9}

The protein-protein interaction network (PPIN) results showed that MMP9 interacts with both LCN2 and LOXL2. To confirm this, the endogenous interactions between three proteins were detected by immunofluorescence (IF). It revealed that in the KYSE150 cell line, LCN2 and LOXL2, LCN2 and MMP9, and LOXL2 and MMP9 were all co-localized, with the Pearson correlation coefficients as 0.79, 0.92 and 0.91, respectively (Fig. 1A). Therefore, the ternary complex of LCN2/LOXL2/MMP9 might exist in esophageal cancer cells. To further confirm the presence of LCN2/LOXL2/MMP9 ternary complexes in ESCC cells, co-immunoprecipitation using whole cell lysates was performed after co-transfection of LCN2-HA/LOXL2-Flag, LCN2-HA/MMP9-Flag, or LOXL2-HA/MMP9-Flag.

Considering LCN2, LOXL2 and MMP9 are all secreted proteins, the culture supernatant after transfection was also collected and concentrated to detect their extracellular interactions. The results showed that both in KYSE410 and KYSE150 cells, protein-protein interaction between LCN2 and LOXL2, and between LCN2 and MMP9, occurred both intracellularly and extracellularly (Fig. 1B-C). Nevertheless, the proteinprotein interactions between LOXL2 and MMP9 occurred only intracellularly (Fig. 1D). Next, we cooverexpressed LCN2-HA/LOXL2-HA/MMP9-Flag in KYSE410 cells and carried out co-immunoprecipitation using the whole cell lysates and culture supernatants. We found protein-protein interactions between MMP9 and LCN2 both intracellularly and extracellularly, whereas the protein-protein interaction between MMP9 and LOXL2 was identified only intracellularly (Fig. 1E). The schematic intracellular and extracellular protein-protein interaction pattern of LCN2/LOXL2/MMP9 is shown in Fig. 1F.

\section{LCN2/LOXL2/MMP9 protein-protein interaction promotes migration and invasion of ESCC cells}

Since LCN2, LOXL2 and MMP9 form a ternary complex in esophageal cancer cells, their biological function regarding the ternary complex remained to be explored. In the KYSE150 cell line, wound healing experiments showed that co-overexpression of LCN2/LOXL2, LCN2/MMP9, and LOXL2/MMP9 increased the migration of ESCC cells (Fig. 2A). Moreover, both transwell assays and invasion assays showed LCN2/LOXL2, LCN2/MMP9, LOXL2 /MMP9 enhanced the migration and invasion of esophageal cancer cells (Fig. 2B-C). Similar results were also obtained with the KYSE410 cell line (Supplementary Fig. S2AC). Compared to the solely transfected LCN2 group, either co-transfection of LCN2/LOXL2 or cotransfection of all three significantly improved the migration and invasion of esophageal cancer cells (Supplementary Fig. S2D).

In addition to migration and invasion, we also investigated the functional role of the LCN2/LOXL2/MMP9 ternary complex in cell proliferation. Colony-forming assays showed that overexpression of LCN2/LOXL2, 
LCN2/MMP9, LOXL2/MMP9 enhanced cell colony formation in the KYSE150 cell line (Fig. 2D). Similar results were obtained with the KYSE410 cell line (Supplementary Fig. S2E). MTS assay results showed that proliferation of ESCC cells in the experimental group did not change significantly after their overexpression in either KYSE410 or KYSE150 cells (Supplementary Fig. S2F-G). In the KYSE150 cell line, flow cytometry showed no significant cell cycle change in the experimental group (Supplementary Fig. $\mathrm{S} 2 \mathrm{H})$.

To obtain a stably overexpressing cell line, the Neon ${ }^{\mathrm{TM}}$ Transfection System (Thermo) was used to electroporate the LCN2-HA/LOXL2-HA/MMP9-Flag (abbreviated as LLM) plasmids into the KYSE150 cell line, followed by two weeks of $\mathrm{G} 418$ selection. Western blotting showed that a cell line stably overexpressing all three proteins was successfully established (Fig. 2E). Consistently, wound healing experiments showed that LLM increased the migration of esophageal cancer cells (Fig. 2F). In order to study the effect of LLM on ESCC tumor progression in vivo, we separately injected the cells stably overexpressing the ternary LLM complex and control cells into the footpads of nude mice. Compared with the control group, LLM cells displayed enhanced tumor progression (Fig. 2G). The anatomy results showed that 4 out 6 nude mice overexpressing LLM developed tumor metastasis, where the tumor cells migrated from the footpad to the ankle, and the lymph nodes of 2 nude mice were swollen (Fig. $2 \mathrm{H}$ ).

\section{LCN2/ LOXL2 and MMP9/ LOXL2 interactions depend on the SRCR domains}

To identify the specific domains for LOXL2/LCN2, and LOXL2/MMP9 protein-protein interaction in the ternary complex, two truncated LOXL2 plasmids were constructed, DLOXL2-HA (545-774aa) (lacking the $\mathrm{N}$-terminal scavenger receptor cysteine-rich (SRCR) domains, denoted D4-HA), and DLOXL2-HA (1-544aa) (lacking the C-terminal amine oxidase domain, denoted D9-HA), respectively. First, these two plasmids were able to be expressed in KYSE150 cells (Fig. 3A). Immunofluorescence showed that in the KYSE150 cell line, D4-HA did not co-localize with either LCN2 or MMP9, with Pearson correlation coefficients of -0.27 and 0.21 , respectively (Fig. 3B). Confocal Z-stack analysis was applied to scan different sections of transfected cells to confirm their co-localization (Supplementary Fig. S3). Further, co-immunoprecipitation results showed that, the protein-protein interactions between LOXL2 and either LCN2 or MMP9 disappeared when the SRCR domains of LOXL2 were deleted (Fig. 3C-D). These results suggested the protein-protein interactions for LCN2/LOXL2 and MMP9/LOXL2 both depended on the SRCR domains of LOXL2. Furthermore, the biological roles of the N-terminus and C-terminus of LOXL2 was investigated. Transwell and invasion assays showed that the two truncated plasmids (D4 and D9) could reduce the ability of ESCC cells to migrate and invade, as well as colonize, compared to full-length LOXL2 (Fig. 3E$\mathrm{G)}$.

\section{LCN2 inhibitor DFOM inhibits the migration and tumor growth of ESCC cells}

Deferoxamine mesylate (DFOM) is an iron chelator that binds free ferric iron and is mainly used to treat diseases caused by iron overload (19). KYSE1 50 cells were treated with 0, 5, 10, 25, 50, 100 and $200 \mu \mathrm{M}$ DFOM. After $48 \mathrm{~h}$, an MTS assay was performed, and the IC50 was calculated to be $30.993 \mu \mathrm{M}$. 
Therefore, $30 \mu \mathrm{M}$ DFOM was used for subsequent experiments. DFOM could decrease the expression level of LCN2 in KYSE150 cells (Fig. 4A). Wound healing experiments showed that LCN2 overexpressionmediated enhancement of ESCC cell migration could be reduced by DFOM treatment (Fig. 4B). Compared with the stable overexpression of LLM, these results show that the expression of LCN2 can be significantly reduced after DFOM treatment, indicating that DFOM may destroy the formation of the ternary complex LLM or reduce its expression (Fig. 4C). Compared with empty vector group, the migration of ESCC cells could be significantly promoted by LLM, which was decreased after DFOM treatment (Fig. 4D). Colony-forming assays found that LCN2 or LLM could promote the proliferation ability of single ESCC cells, which could be inhibited by DFOM treatment (Fig. 4E). In order to study the effect of LCN2 and the LLM ternary complex on tumor progression of ESCC in vivo, as well as the anti-tumor effect of DFOM in vivo, a xenograft model was applied to observe and evaluate. Compared with the control group, LCN2 promoted ESCC tumor growth in vivo. But after intraperitoneal administration of DFOM, it was found that both the tumor volume and weight were significantly reduced (Fig. 4F). Similarly, the LLM ternary complex promoted tumor progression in vivo compared to the vector group. Consistently, tumor growth was inhibited, and the tumor volume and weight were also reduced after DFOM treatment (Fig. $4 G)$.

\section{LCN2/LOXL2/MMP9 promote the degradation of ECM}

The LCN2/LOXL2/MMP9 ternary complex can promote the migration and invasion of ESCC cells, but the specific molecular mechanism is still unclear. In the experimental groups that overexpressed LCN2/MMP9 or LOXL2/MMP9, the ability to degrade gelatin for MMP9 was elevated in zymography experiments (Fig. 5A). It was also observed that gelatin degradation at $130 \mathrm{kD}$ occurred for extracts of LCN2/MMP9 co-overexpressing cells, which was corresponding to a LCN2/MMP9 complex. In cells cooverexpressing LCN2/MMP9 or LOXL2/MMP9, gelatin degradation was also seen at around 200kD, which coincides with the predicted molecular weight of the LCN2/LOXL2/MMP9 ternary complex. We presumed that when co-overexpressing LCN2/MMP9 or LOXL2/MMP9, a LCN2/LOXL2/MMP9 ternary complex synergistically degrades the matrix, promoting the migration and invasion of ESCC cells (Fig. $5 A)$.

Next, visualization of ECM degradation in living cells was carried out using fluorescent matrix degradation assays. In KYSE150 cells co-overexpressing LCN2/LOXL2, LCN2/MMP9 and LOXL2/MMP9, the ability to degrade fluorescent Matrigel was enhanced, as a significant black ghost was formed after degradation, whereas Matrigel degradation was barely observed in control cells (Fig. 5B). Three sets of co-overexpressing cells were scanned from top to bottom using the Z-stack function of the confocal microscope. The results confirmed that cells co-overexpressing LCN2/LOXL2, LCN2/MMP9 or LOXL2/MMP9 degraded fluorescent Matrigel around the cell spheroids (Supplementary Fig. S4).

The growth environment of tumor cells in vivo is three-dimensional. For this reason, we further characterized the ability of tumor cells to degrade ECM in a 3D cell co-culture model. Cells in the control group displayed a round and smooth morphological structure, except for the enlargement of the cell body 
itself. In the LCN2/LOXL2-, LCN2/MMP9- or LOXL2/MMP9-overexpressing groups, in addition to enlargement of the cell body, the ability of cells to degrade Matrigel was enhanced as more Matrigel surrounding the cells was digested. With the prolongation of culture time, elongation and branching of filopodia around the cells became clearly visible (Fig. 5C). In the stably overexpressing LCN2/LOXL2/MMP9 cell line, a similar result was obtained that LLM can significantly degrade Matrigel and promote the extension of filopodia (Fig. 5D).

MMP9 activity in the ternary complex was examined. The zymography results indicated that the ternary complex LLM could significantly enhance the enzymatic activity of MMP9 to degrade gelatin and form a distinct degradation band, while DFOM treatment could significantly weaken the enzymatic activity of MMP9 (Fig. 5E). The results of 3D cell co-culture found that after DFOM treatment of ESCC cells, the ability of the LLM complex to degrade Matrigel was reduced along with the number of filopodia (Fig. 5F).

Since the interaction of LCN2/LOXL2 and MMP9/LOXL2 depends on the SRCR domains of LOXL2, we further detected the importance of the SRCR domains. The formation of filopodia was observed in cells transfected with truncated LOXL2 and the full-length LOXL2 after $48 \mathrm{~h}$ (Supplementary Fig. S5). The number and extension length of filamentous pseudopods of cells transfected with full-length LOXL2 were the most prominent, compared to those in cells transfected with the catalytic domain (D4) (Supplementary Fig. S5). Minimal change was observed in cells transfected with the SRCR domain (D9), indicating that the catalytic domain of LOXL2 plays an important role in ECM degradation and filopodia formation (Supplementary Fig. S5).

\section{LCN2/LOXL2/MMP9 induce cytoskeletal microfilament remodeling}

Tumor cell migration and invasion usually involve rearrangement of the cellular microfilament system. In order to better observe changes in the actin cytoskeleton of ESCC cells, F-actin in KYSE410 cells was fluorescently labeled after the overexpression of target proteins. The results showed that stress fibers in control cells were arranged in regular bundles, while the stress fibers in the overexpression group were more disordered, suggesting that overexpression of LCN2, LOXL2 and MMP9 could promote cell migration by remodeling the cell microfilament cytoskeleton (Fig. 6A). Profilin 1 (PFN1) is a member of the profilin family of small actin-binding proteins that play an important role in actin dynamics by regulating actin polymerization in response to extracellular signals [20]. To observe the actin arrangement, F-actin and profilin 1 were simultaneously fluorescence labeled after LCN2/LOXL2, LCN2/MMP9 and LOXL2/MMP9 were transfected. The microfilament skeleton in the three overexpressing groups was more disordered, compared with the control group (Fig. 6B). The protein level of profilin 1 was increased and accumulated in the cytoplasm and at the leading edge of the cell. These results indicated that LCN2/LOXL2/MMP9 promotes cell migration by enhancing profilin 1 expression and promoting microfilament rearrangement (Fig. 6B).

In the KYSE410 cell line, LCN2-HA/LOXL2-HA/MMP9-Flag was overexpressed, and the HA and Flag tags were fluorescently labeled. It could be observed that LCN2, LOXL2, and MMP9 were all successfully overexpressed. Compared with the empty vector group, the cytoskeleton of cells overexpressing LLM was 
disordered, and the number of filopodia was significantly increased (Fig. 6C). Next, we aimed to analyze whether DFOM treatment could rescue the cytoskeleton disorder. We found the fluorescence intensity of profilin1 increased in LLM-overexpressing cells, but was decreased after DFOM treatment and accompanied by restoration of the cytoskeleton organization to an orderly state (Fig. 6D). These results suggest that the LLM ternary complex may enhance the expression of profilin 1 . To confirm this, western blotting showed the expression of profilin 1 was decreased after DFOM treatment (Fig. 6E).

\section{LCN2/LOXL2/MMP9 activate the FAK/AKT/GSK3 $\beta$ signaling pathway}

Next, we wanted to identify the signaling pathways involving the LLM. After LCN2, LOXL2 and MMP9 were overexpressed individually, the phosphorylation levels of NFkB, STAT3, and PTEN remained unchanged, while phosphorylation level of AKT increased, indicating this signaling pathway was activated (Fig. 7A). After co-overexpression of LCN2/LOXL2, LCN2/MMP9 and LOXL2/MMP9, the phosphorylation levels of NFkB, STAT3 and PTEN still remained unchanged, while the phosphorylation levels of FAK, AKT and GSK3 $\beta$ also increased, suggesting that LCN2/LOXL2/MMP9 protein-protein interaction promotes migration and invasion of ESCC cells by activating the FAK/AKT/GSK3 $\beta$ signaling pathway (Fig. 7B). Similar alternations were also observed in KYSE410 cells (Fig. 7C). On the other hand, co-overexpression of LCN2/LOXL2, LCN2/MMP9 or LOXL2/MMP9 induced no significant change the level of phosphorylated ERK (Supplementary Fig. S6).

For further confirmation, pathway activators or inhibitors were applied to confirm the involvement of these signaling pathways. Capivasertib (MCE, HY-15431), which increases phosphorylation of AKT at both Ser473 and Thr308 (21), was added to KYSE150 cells to mimic the activation by

LCN2/LOXL2/MMP9 complex. Compared with the control group, AKT phosphorylation was enhanced by capivasertib, and the expression level of LCN2, LOXL2 and MMP9 were increased as well (Fig. 7D). Wortmannin is a pan-inhibitor of PI3K. When cells overexpressing LCN2/LOXL2/MMP9 were grown mixed with Matrigel in a 3D model to observe the changes of cell morphology, no obvious changes were observed in filopodia number and length except the enlarge of cell body itself. However, filopodia in the overexpression group began to form at $3 \mathrm{~h}$ after transfection. After $6 \mathrm{~h}$, most cells in overexpressing group had formed filopodia that extended around the cell surface, thus promoting cell spreading, morphological change and cell migration. On the other hand, LCN2/LOXL2/MMP9-overexpressing cells treated with wortmannin had barely extended filopodia after $6 \mathrm{~h}$, and only part of cells began to form the short filamentous pseudopods (Fig. 7E).

The SPOCK1 gene encodes a matricellular glycoprotein that belongs to a family of novel Ca2+-binding proteoglycans that promote cell migration in many tumors [22]. Expression of SPOCK1 was clearly increased after LCN2/LOXL2/MMP9 overexpression, to reshape the ECM, then promote the migration and invasion of ESCC cells. Next, the PI3K inhibitor wortmannin was added to cells overexpressing LCN2/LOXL2/MMP9. Wortmannin treatment resulted in inhibition of SPOCK1 expression, suggesting that the ternary complex of LCN2/LOXL2/MMP9 activates the AKT signaling pathway to promote SPOCK1 expression (Fig. 7F). Interestingly, overexpression of SPOCK1 in KYSE150 cells enhanced 
phosphorylation of AKT, suggesting a positive regulation feed-back loop between SPOCK1 and p-AKT involved by LCN2/LOXL2/MMP9 (Fig. 7G).

\section{Discussion}

Dysregulation of ECM structure and components are key events in tumor progression. The ability of cancer cells to migrate and invade is one of the hallmarks of solid metastatic cancer [23]. It is critical to understand how cancer cells interact with their microenvironment to migrate and invade the surrounding tissue, move towards the vasculature (blood/lymphatic vessels), and extravasate to create distant metastases, to discovering efficient targets for anti-cancer therapy (24).

Numerous studies have shown that LCN2, a secreted protein, is closely related to the malignant progression of tumors. Elevated LCN2 expression has been observed in various human solid tumors, including breast, colorectal, ovarian, gastric, ovarian, bladder, kidney, lung cancers, and ESCC [9]. A higher expression level of LCN2 is usually associated with tumor size, tumor stage and invasion of carcinoma cells, serving as a poor prognostic factor. These characteristics strongly suggest LCN2 might be a potential biomarker and therapeutic target in malignancies [9]. Previous studies have reported that LCN2 and MMP9 can form a heterodimer through disulfide bonding to protect MMP9 enzyme activity, contributing greatly to tumor invasion and metastasis [11,25-26]. High levels of monomeric forms of LCN2 and MMP-9, and LCN2-MMP-9 heterodimers are secreted into the extracellular space, and their levels seem to correlate with the aggressive behavior of neoplastic cells in several types of cancer (9). On the other hand, LOXL2 can effectively enhance MMP9 enzyme activity by elevating MMP9 expression [27]. LOXL2 could catalyze the cross-linkage of extracellular collagen to change the stiffness of the ECM, facilitating the motility of cancer cells [27-28]. MMP9 plays a core role in the degradation of the extracellular matrix and basement membrane of cancers to contribute to tumor invasion and metastasis [29-30].

Considering the crosstalk of functional roles for LCN2, MMP9 and LOXL2 in tumor progression, we hypothesized that an LCN2/LOXL2/MMP9 ternary complex might exist in esophageal cancer and play a synergistic role in biological function. First, we used mass spectroscopy to show LCN2 could directly interact with LOXL2. An LCN2/LOXL2 PPI network suggested a physical interaction might occur between LCN2 and LOXL2, which might also involve other interacting proteins, such as MMP9. Our results show that an LCN2/LOXL2/MMP9 ternary complex is formed in esophageal cancer, with a distinctive intracellular and extracellular interaction pattern. The SRCR structure is a key domain through which LOXL2 interacts with both LCN2 and MMP9 to play an important biological role in esophageal cancer. In recent years, the N-terminal SRCR repeats for the LOX gene family has been found to play an important functional role [11]. Similarly, the SRCR domain in LOXL3, rather than the C-terminal oxidase catalytic domain, represents the major deacetylase/deacetyliminase activity in the modification of STAT3 [31].

Based on LCN2/LOXL2, LCN2/MMP9, LOXL2/MMP9 protein-protein interactions, we further elucidated the molecular and cellular mechanisms underlying the LCN2/LOXL2/MMP9 ternary complex on 
promoting migration and invasion of esophageal cancer cells, playing a synergistic role. Zymography showed that an elevated LCN2/LOXL2/MMP9 ternary complex was presented, especially in the case of enhanced expression of MMP9. MMP9 has three fibronectin type II homologous repeat domains that bind to gelatin with high affinity, making gelatin the major substrate for MMP9 (32-33). Degradation of fluorescent fibronectin (FN) experiments revealed that co-expression of LCN2/LOXL2, LCN2/MMP9, or LOXL2/MMP9 confers a greater ability to degrade FN. FN is a major non-collagen glycoprotein in the extracellular matrix and basement membrane, which has the function of adhering fibrin and collagen [34]. A previous study found a high stromal FN content facilitates tumor cell metastasis by promoting morphological change and improving the motility and migratory ability of ESCC cells [35]. Therefore, the LCN2/LOXL2/MMP9 ternary complex can promote the invasion of esophageal cancer cells by elevating the expression of MMPs to degrade gelatin and fibronectin. Considering that growth of tumor cells in vivo occurs in a three-dimensional environment, 3D cell co-culture showed that the LCN2/LOXL2/MMP9 ternary complex degrades the extracellular matrix by promoting the formation and extension of filopodia, thereby enhancing the invasion of tumor cells. Matrigel ${ }^{\circledR}$ is widely used in cancer cell 3D cell co-culture models, which contains proteins commonly found in the basement membrane of epithelial structures, such as laminin, as well as type IV collagen and heparan sulfate proteoglycan [36-37]. MMP9 is able to degrade the laminin and type IV collagen in Matrigel. Therefore, we conclude that the LCN2/LOXL2/MMP9 ternary complex can promote the invasion of cancer cells by degrading laminin and type IV collagen.

In addition to degradation of the extracellular matrix, the mobility of tumor cells is also an important factor contributing to migration and invasion. Actin is a highly conserved protein that participates in various types of cell movement and is universally expressed in all eukaryotic cells. Polymerization of individual actin filaments into organized parallel bundles occurs in filopodia, which are slender protrusions that can extend far beyond the cell edge and reach or sense distant targets [38]. Profilin 1 (PFN1) is an abundant actin-binding protein that promotes nucleotide exchange of actin and converts ADP/G-actin to ATP/G-actin [39]. The PFN1-ATP/G-actin complex can interact with the fast-growing end of F-actin to increase ATP/G-actin for growing actin filaments. Thus, profilin 1 is a central mediator of actin microfilament and microtubule dynamics [20]. Cells overexpressing LCN2/LOXL2, LCN2/MMP9, LOXL2/MMP9 or LLM exhibit a disordered microfilament skeleton, increased filopodia, and increased expression of profilin 1 distributed in the cell interior and the leading edge of the cell. These results suggest that LCN2/LOXL2/MMP9 could enhance cell migration by promoting microfilament rearrangement and enhancing profilin 1 expression. The cytoskeleton, with its regulatory and structural proteins, had emerged as a novel and highly effective target to be exploited for specific anti-metastatic drugs [40]. Though at a glance, it is contradictory that when considering their individual role in ECM remodeling, LCN2//MMP9 mainly contributes to matrix degradation, while LOXL2 strengthens ECM stiffness by catalyzing the cross-linking of collagen and elastin. Nevertheless, we provide evidence that LCN2/LOXL2, LCN2/MMP9, LOXL2/MMP9 or LLM affect tumor growth and progression in a synergistic manner. We presumed that ECM degradation opens a path, and ECM stiffness favors the assembly of filopodia and invadosome, both of which contribute to cancer cell migration and invasion. 
Iron (Fe) plays a vital role in various biological processes. Cancer cells show a high rate of Fe metabolism and therefore require more Fe to proliferate. Evidence indicates that tumor cells are very sensitive to iron deficiency, much more sensitive than normal cells [41]. LCN2 is an important ferritin carrier, which can increase the iron level in cells to promote the progression of malignant behavior [42]. Fe chelating agents have been used to treat a variety of diseases, such as leukemia, neuroblastoma and breast cancer [43]. Among them, DFOM has been extensively studied and found to have anti-tumor effects by the treatment of iron overload [44-45]. In this study, we analyzed the effect of DFOM in ESCC tumor progression by targeting LCN2, and the LCN2/LOXL2/MMP9 complex as well. We show that DFOM inhibits the migration and invasion of esophageal cancer cells through multiple ways, including reducing the expression of LCN2 and LLM, suppressing MMP9 enzyme activity, inhibiting cytoskeletal rearrangement, and thus restoration of ECM remodeling. Furthermore, our in vivo experiments show that DFOM can inhibit ESCC tumor growth.

Leng et al. found that AKT signaling up-regulates LCN2 expression and promotes breast cancer invasion and migration [46]. It has been reported that LCN2 activates the PI3K/AKT pathway in smooth muscle cells [47]. In endothelial cells, LOXL2 activates AKT and FAK signaling, which participate in the regulation of EMT [48]. Zheng et al. found that MMP9 promotes the occurrence of lung cancer through the PI3K/AKT signaling pathway [49]. In this study, we found that the LCN2/LOXL2/MMP9 ternary complex activates the FAK/AKT/GSK3 $\beta$ signaling pathway to promote expression of SPOCK1. SPOCK1 is closely related to cell proliferation, adhesion and metastasis, and it is highly expressed in a variety of cancers, including esophageal squamous cell carcinoma, liver, gallbladder, colon, and prostate cancer [50]. Liu et al. found that SPOCK1 upregulates the expression and activity of MMP9, causes remodeling of the ECM and promotes tumor cell migration and invasion [51]. Therefore, LCN2/LOXL2/MMP9 activates the FAK/AKT/GSK3 $\beta$ signaling pathway to enhance SPOCK1 expression and remodel ECM, thus promoting the migration and invasion of esophageal cancer cells. Moreover, degradation of the ECM could be inhibited after adding the PI3K inhibitor wortmannin, suggesting that inhibition of AKT phosphorylation at ser473 could inhibit the migration and invasion of esophageal cancer. So, AKT (ser473) phosphorylation could be applied as a potential therapeutic target for esophageal cancer. Taken together, we propose a schematic model that the LCN2/LOXL2/MMP9 complex promotes the migration and invasion of cancer cells, through intracellular and extracellular means (Fig. 8).

\section{Conclusions}

In this study, we found that an LCN2/LOXL2/MMP9 ternary complex can activate the FAK/AKT/GSK3 $\beta$ signaling pathway, degrade extracellular matrix components such as FN and collagen IV, remodel the cytoskeleton, and enhance the expression of profilin 1 and SPOCK 1 to promote the migration and invasion of esophageal cancer cells, and ultimately lead to malignant progression of esophageal cancer. On the other hand, DFOM can inhibit the progression of esophageal cancer. LCN2, LOXL2 and MMP9 might serve as promising therapeutic targets in solid tumors including ESCC. 


\section{Abbreviations}

ECM: extracellular matrix; LOXL2: lysyl oxidase-like 2; MMP9: matrix metalloproteinase 9; LCN2: lipocalin

2; DFOM: deferoxamine mesylate; ESCC: esophageal squamous cell carcinoma; MMPs: Matrix metalloproteinases; NGAL: neutrophil gelatinase-associated lipocalin; EMT: epithelial-mesenchymal transformation.

\section{Declarations}

\section{Ethics approval and consent to participate}

N/A - No patients were not involved in this study.

\section{Consent for publication}

N/A - The manuscript has no individual person's data in any form.

\section{Availability of data and materials}

The datasets used and/or analyzed during the current study are available from the corresponding author on reasonable request.

Competing interests

The authors declare that they have no competing interests.

\section{Funding}

This work was supported by grants from the National Natural Science Foundation of China (No. 81672473), the Science and Technology Program of Guangdong (No. 2017A03031381).

\section{Author contributions}

BW, EL and ZD conceived and supervised the project, designed experiments. QX, MC, XZ, WB, LL, YZ, JD, $X Z$ performed investigation, data analyses. LX supervised the project. $\mathrm{HZ}$. and SW carried out project administration. QX wrote the original draft. BW, EL and ZD modified the manuscript. BW acquired funding. All authors read and approved the final manuscript.

\section{Acknowledgments}

We thank Dr. Stanley Lin from Shantou University Medical College for proof-reading of the manuscript.

\section{References}

1. Sung H, Ferlay J, Siegel RL, Laversanne M, Soerjomataram I, Jemal A, et al. Global cancer statistics 2020: GLOBOCAN estimates of incidence and mortality worldwide for 36 cancers in 185 countries. 
CA Cancer J Clin. 2020;70:313.

2. He H, Chen N, Hou Y, Wang Z, Zhang Y, Zhang G, et al. Trends in the incidence and survival of patients with esophageal cancer: A SEER database analysis. Thorac Cancer. 2020;11:1121-8.

3. Cao W, Chen HD, Yu YW, Li N, Chen WQ. Changing profiles of cancer burden worldwide and in China: a secondary analysis of the global cancer statistics 2020. Chin Med J (Engl). 2021;134:783-91.

4. Kai F, Drain AP, Weaver VM. The Extracellular Matrix Modulates the Metastatic Journey. Dev Cell. 2019;49:332-46.

5. Cabral-Pacheco GA, Garza-Veloz I, Castruita-De la Rosa C, Ramirez-Acuña JM, Perez-Romero BA, Guerrero-Rodriguez JF, et al. The Roles of Matrix Metalloproteinases and Their Inhibitors in Human Diseases. Int J Mol Sci. 2020;21:9739.

6. Rodvold JJ, Mahadevan NR, Zanetti M. Lipocalin 2 in cancer: when good immunity goes bad. Cancer Lett. 2012;316:132-8.

7. Hu C, Yang K, Li M, Huang W, Zhang F, Wang H. Lipocalin 2: a potential therapeutic target for breast cancer metastasis. Onco Targets Ther. 2018;11:8099-106.

8. Yan L, Borregaard N, Kjeldsen L, Moses MA. The high molecular weight urinary matrix metalloproteinase (MMP) activity is a complex of gelatinase B/MMP-9 and neutrophil gelatinaseassociated lipocalin (NGAL). Modulation of MMP-9 activity by NGAL. J Biol Chem. 2001;276:3725865.

9. Santiago-Sánchez GS, Pita-Grisanti V, Quiñones-Díaz B, Gumpper K, Cruz-Monserrate Z, Vivas-Mejía PE. Biological Functions and Therapeutic Potential of Lipocalin 2 in Cancer. Int J Mol Sci. 2020;21:4365.

10. Du ZP, Lv Z, Wu BL, Wu ZY, Shen JH, Wu JY, et al. Neutrophil gelatinase-associated lipocalin and its receptor: independent prognostic factors of oesophageal squamous cell carcinoma. J Clin Pathol. 2011;64:69-74.

11. Du ZP, Wu BL, Xie YM, Zhang YL, Liao LD, Zhou F, et al. Lipocalin 2 promotes the migration and invasion of esophageal squamous cell carcinoma cells through a novel positive feedback loop. Biochim Biophys Acta. 2015;1853:2240-50.

12. Vallet SD, Ricard-Blum S. Lysyl oxidases: from enzyme activity to extracellular matrix cross-links. Essays Biochem. 2019;63:349-64.

13. Li TY, Xu LY, Wu ZY, Liao LD, Shen JH, Xu XE, et al. Reduced nuclear and ectopic cytoplasmic expression of lysyl oxidase-like 2 is associated with lymph node metastasis and poor prognosis in esophageal squamous cell carcinoma. Hum Pathol. 2012;43:1068-76.

14. Zhan XH, Jiao JW, Zhang HF, Xu XE, He JZ, Li RL, et al. LOXL2 Upregulates Phosphorylation of Ezrin to Promote Cytoskeletal Reorganization and Tumor Cell Invasion. Cancer Res. 2019;79:4951-64.

15. Wen B, Xu LY, Li EM. LOXL2 in cancer: regulation, downstream effectors and novel roles. Biochim Biophys Acta Rev Cancer. 2020;1874(2):188435. 
16. Barillari G. The Impact of Matrix Metalloproteinase-9 on the Sequential Steps of the Metastatic Process. Int J Mol Sci. 2020;21:4526.

17. Zou H, Wen B, Li RL, Zhan XH, Jiao JW, Liao LD, et al. Lysyl oxidase-like 2 promotes esophageal squamous cell carcinoma cell migration independent of catalytic activity. Int J Biochem Cell Biol. 2020;125:105795.

18. Du Z, Wu B, Xia Q, Zhao Y, Lin L, Cai Z, et al. LCN2-interacting proteins and their expression patterns in brain tumors. Brain Res. 2019;1720:146304.

19. Komoto K, Nomoto T, El Muttaqien S, Takemoto H, Matsui M, Miura Y, et al. Iron chelation cancer therapy using hydrophilic block copolymers conjugated with deferoxamine. Cancer Sci. 2021;112:410-21.

20. Pinto-Costa R, Sousa MM. Profilin as a dual regulator of actin and microtubule dynamics. Cytoskeleton (Hoboken). 2020;77:76-83.

21. Davies BR, Greenwood H, Dudley P, Crafter C, Yu DH, Zhang J, et al. Preclinical pharmacology of AZD5363, an inhibitor of AKT: pharmacodynamics, antitumor activity, and correlation of monotherapy activity with genetic background. Mol Cancer Ther. 2012;11:873-87.

22. Ye Z, Chen J, Hu X, Yang S, Xuan Z, Lu X, et al. SPOCK1: a multi-domain proteoglycan at the crossroads of extracellular matrix remodeling and cancer development. Am J Cancer Res. 2020;10:3127-37.

23. Chaffer CL, Weinberg RA. A perspective on cancer cell metastasis. Science. 2011;331:1559-64.

24. Liu Q, Zhang H, Jiang X, Qian C, Liu Z, Luo D. Factors involved in cancer metastasis: a better understanding to "seed and soil" hypothesis. Mol Cancer. 2017;16:176.

25. Chung IH, Wu TI, Liao CJ, Hu JY, Lin YH, Tai PJ, et al. Overexpression of lipocalin 2 in human cervical cancer enhances tumor invasion. Oncotarget. 2016;7:11113-26.

26. Kobara H, Miyamoto T, Suzuki A, Asaka R, Yamada Y, Ishikawa K, et al. Lipocalin2 enhances the matrix metalloproteinase-9 activity and invasion of extravillous trophoblasts under hypoxia. Placenta. 2013;34(11):1036-43.

27. Wu S, Zheng Q, Xing X, Dong Y, Wang Y, You Y, et al. Matrix stiffness-upregulated LOXL2 promotes fibronectin production, MMP9 and CXCL12 expression and BMDCs recruitment to assist premetastatic niche formation. J Exp Clin Cancer Res. 2018;37:99.

28. López-Jiménez AJ, Basak T, Vanacore RM. Proteolytic processing of lysyl oxidase-like-2 in the extracellular matrix is required for crosslinking of basement membrane collagen IV. J Biol Chem. 2017;292:16970-82.

29. Pittayapruek P, Meephansan J, Prapapan O, Komine M, Ohtsuki M. Role of Matrix Metalloproteinases in Photoaging and Photocarcinogenesis. Int J Mol Sci. 2016;17:868.

30. Yin P, Su Y, Chen S, Wen J, Gao F, Wu Y, et al. MMP-9 Knockdown Inhibits Oral Squamous Cell Carcinoma Lymph Node Metastasis in the Nude Mouse Tongue-Xenografted Model through the RhoC/Src Pathway. Anal Cell Pathol (Amst). 2021;2021:6683391. 
31. Ma L, Huang C, Wang XJ, Xin DE, Wang LS, Zou QC, et al. Lysyl Oxidase 3 Is a Dual-Specificity Enzyme Involved in STAT3 Deacetylation and Deacetylimination Modulation. Mol Cell. 2017;65:296309.

32. Ugarte-Berzal E, Vandooren J, Bailon E, Opdenakker G, Garcia-Pardo A. Inhibition of MMP-9dependent Degradation of Gelatin, but Not Other MMP-9 Substrates, by the MMP-9 Hemopexin Domain Blades 1 and 4. J Biol Chem. 2016;291:11751-60.

33. Van Doren SR. Matrix metalloproteinase interactions with collagen and elastin. Matrix Biol. 2015;44$46: 224-31$.

34. Lin TC, Yang CH, Cheng LH, Chang WT, Lin YR, Cheng HC. Fibronectin in Cancer: Friend or Foe. Cells. 2019;9:27.

35. Xiao J, Yang W, Xu B, Zhu H, Zou J, Su C, et al. Expression of fibronectin in esophageal squamous cell carcinoma and its role in migration. BMC Cancer. 2018;18:976.

36. Anguiano M, Castilla C, Maška M, Ederra C, Peláez R, Morales X, et al. Characterization of threedimensional cancer cell migration in mixed collagen-Matrigel scaffolds using microfluidics and image analysis. PLoS One. 2017;12:e0171417.

37. Kleinman HK, Martin GR. Matrigel: basement membrane matrix with biological activity. Semin Cancer Biol. 2005;15:378-86.

38. Svitkina T. The Actin Cytoskeleton and Actin-Based Motility. Cold Spring Harb Perspect Biol. 2018;10:a018267.

39. Pinto-Costa R, Sousa MM. Profilin as a dual regulator of actin and microtubule dynamics. Cytoskeleton (Hoboken). 2020;77:76-83.

40. Ruggiero C, Lalli E. Targeting the cytoskeleton against metastatic dissemination. Cancer Metastasis Rev. 2021;40(1):89-140.

41. Lelièvre P, Sancey L, Coll JL, Deniaud A, Busser B. Iron Dysregulation in Human Cancer: Altered Metabolism, Biomarkers for Diagnosis, Prognosis, Monitoring and Rationale for Therapy. Cancers (Basel). 2020;12:3524.

42. Torti SV, Torti FM. Iron and cancer: more ore to be mined. Nat Rev Cancer. 2013;13:342-55.

43. Heath JL, Weiss JM, Lavau CP, Wechsler DS. Iron deprivation in cancer-potential therapeutic implications. Nutrients. 2013;5:2836-59.

44. Goto W, Kashiwagi S, Asano Y, Takada K, Morisaki T, Takahashi K, et al. Inhibitory effects of iron depletion plus eribulin on the breast cancer microenvironment. BMC Cancer. 2020;20(1):1215.

45. Bajbouj K, Shafarin J, Hamad M. High-Dose Deferoxamine Treatment Disrupts Intracellular Iron Homeostasis, Reduces Growth, and Induces Apoptosis in Metastatic and Nonmetastatic Breast Cancer Cell Lines. Technol Cancer Res Treat. 2018;17:1533033818764470.

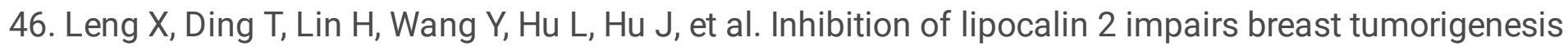
and metastasis. Cancer Res. 2009;69:8579-84. 
47. Wang G, Ma N, Meng L, Wei Y, Gui J. Activation of the phosphatidylinositol 3-kinase/Akt pathway is involved in lipocalin-2-promoted human pulmonary artery smooth muscle cell proliferation. Mol Cell Biochem. 2015;410:207-13.

48. de Jong OG, van der Waals LM, Kools FRW, Verhaar MC, van Balkom BWM. Lysyl oxidase-like 2 is a regulator of angiogenesis through modulation of endothelial-to-mesenchymal transition. J Cell Physiol. 2019;234:10260-9.

49. Zheng H, Liu JF. Studies on the relationship between P13K/AKT signal pathway-mediated MMP-9 gene and lung cancer. Eur Rev Med Pharmacol Sci. 2017;21:753-9.

50. Sun LR, Li SY, Guo QS, Zhou W, Zhang HM. SPOCK1 Involvement in Epithelial-to-Mesenchymal Transition: A New Target in Cancer Therapy? Cancer Manag Res. 2020;12:3561-9.

51. Liu YZ, Guo YF, Wang L, Tan LJ, Liu XG, Pei YF, et al. Genome-wide association analyses identify SPOCK as a key novel gene underlying age at menarche. PLoS Genet. 2009;5:e1000420.

\section{Figures}



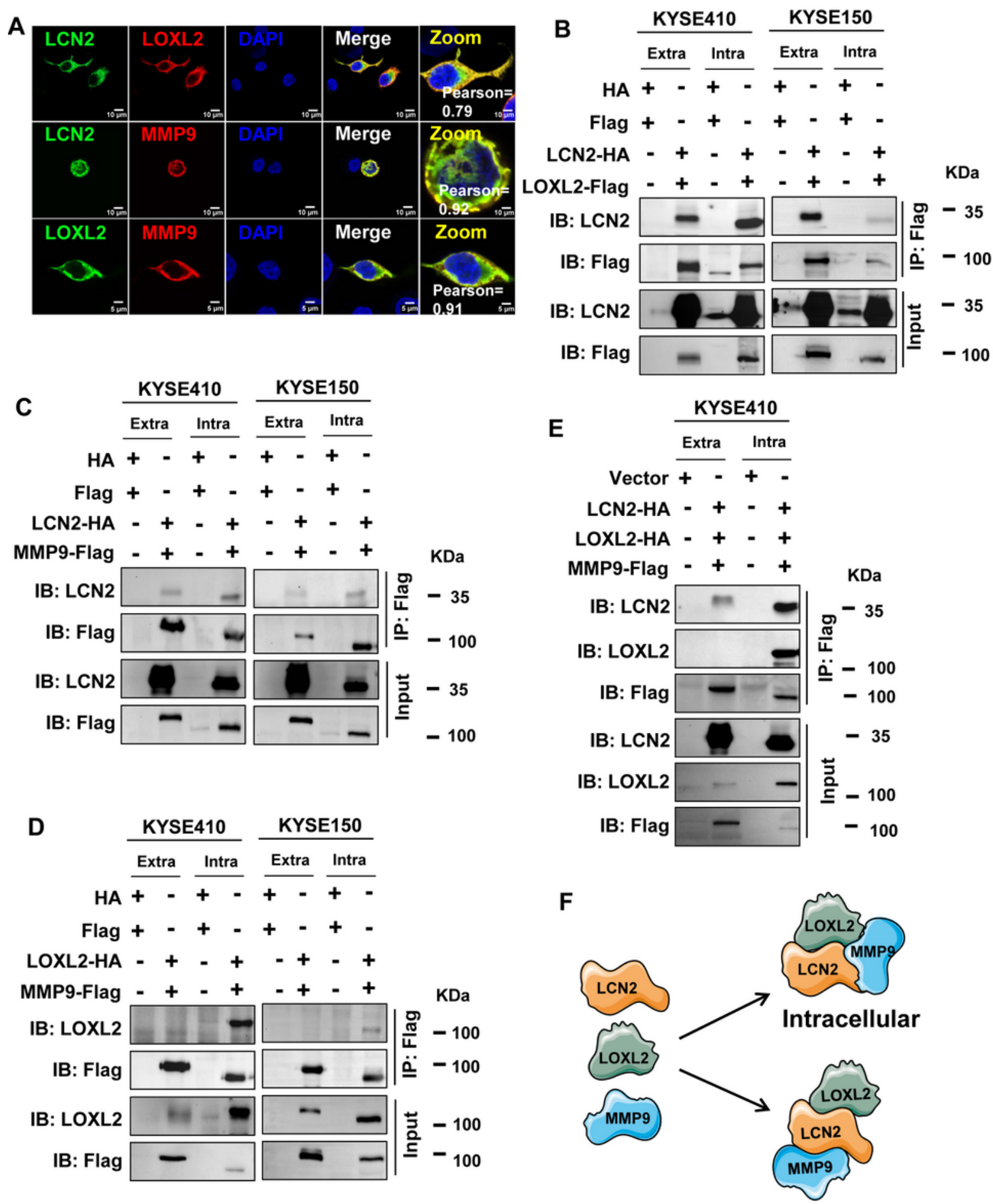

Extracellular

Figure 1

Identification of LCN2/LOXL2/MMP9 protein-protein interaction patterns. (A) Immunofluorescence was applied to observe the endogenous co-localization of LCN2 with LOXL2, LCN2 with MMP9, and LOXL2 with MMP9, respectively, in the KYSE150 cell line. For the interaction experiments, several pairs of plasmids were co-transfected into KYSE410 and KYSE150 cells, LCN2-HA/LOXL2-Flag (B), LCN2HA/MMP9-Flag (C), LOXL2-HA/MMP9-Flag (D). Co-IP was used to detect the interaction between LCN2- 
HA / LOXL2-HA / MMP9-Flag in KYSE410 (E). After $48 \mathrm{~h}$ of transfection, total protein was extracted and the cell-conditioned medium was concentrated, followed by co-IP with the indicated antibodies. (F) Schematic diagram of the intracellular and extracellular protein-protein interactions between LCN2/LOXL2/MMP9.
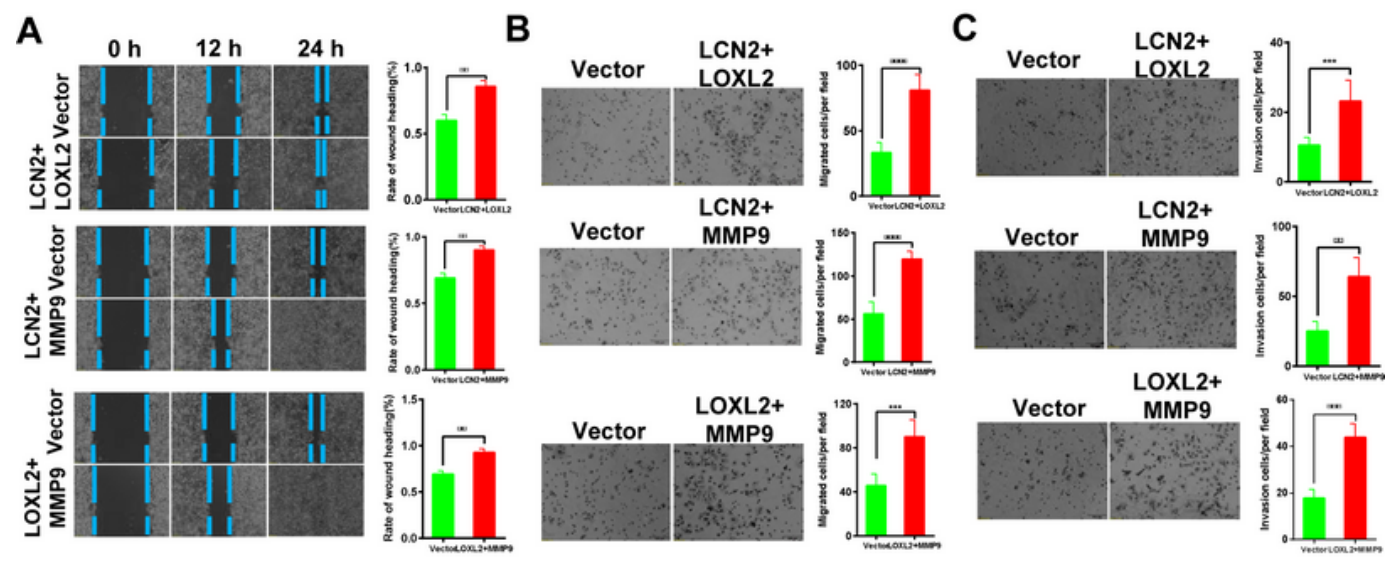

D
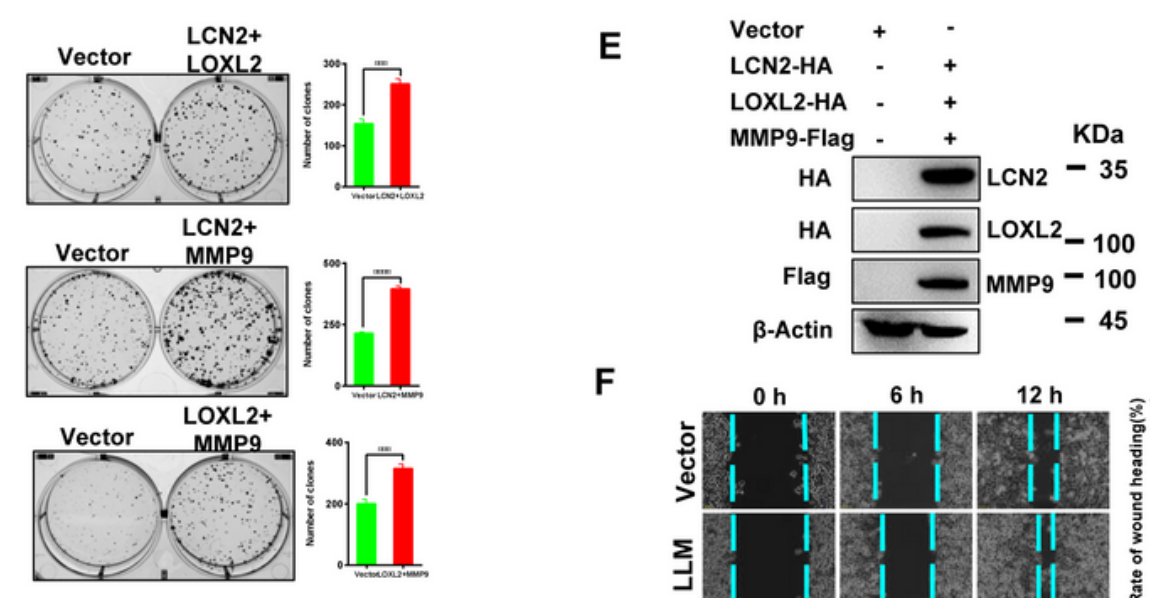

$\mathbf{F}$

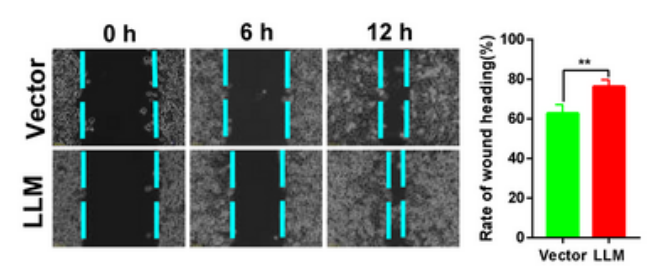

G
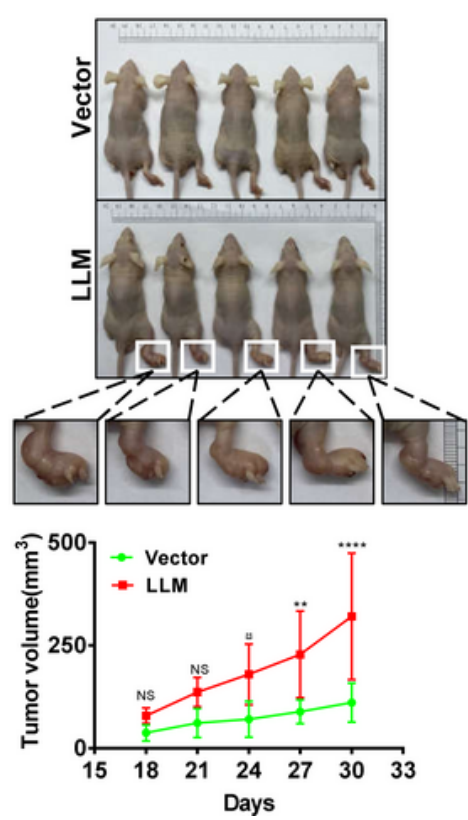

H

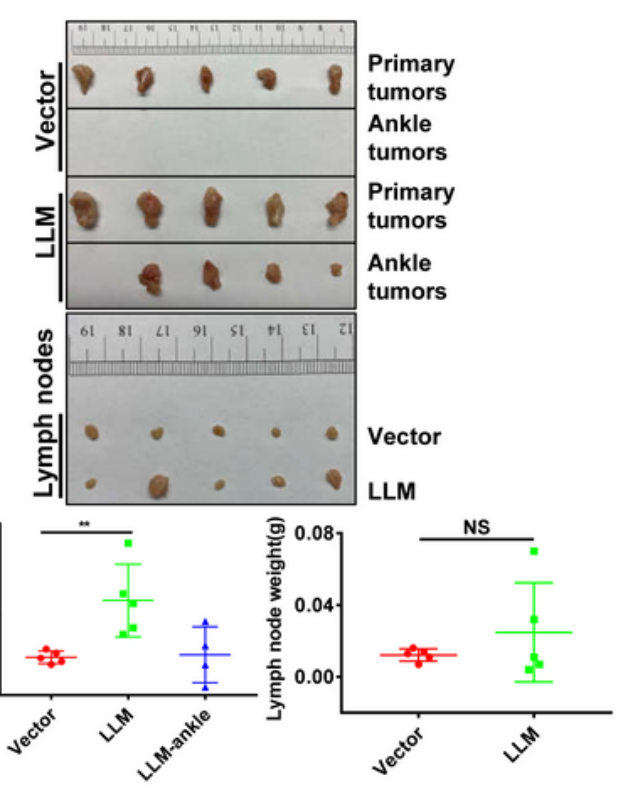

Figure 2 
Protein-protein interaction between LCN2/LOXL2/MMP9 promotes migration and invasion of ESCC cells. LCN2/LOXL2, LCN2/MMP9, or LOXL2/MMP9 were co-transfected into KYSE150 cells. Wound-healing (A) and migration assays (B) were used to detect the migration of esophageal cancer cells. (C) A transwell assay with Matrigel-coated membranes was applied to study the invasive capacity of ESCC cells. (D) Colony-formation assays were used to detect the proliferation of ESCC cells. (E) Western blotting verified the stable expression of LCN2-HA/LOXL2-HA/MMP9-Flag in KYSE150 cells. (F) Migration of esophageal cancer cells was detected by wound-healing assay in the LCN2/LOXL2/MMP9-overexpressing KYSE150 cell line. (G) $100 \mu$ l of KYSE150 $(1 \times 107$ cells/ml in serum-free medium) stably expressing LCN2/LOXL2/MMP9 or empty vector was injected into the footpads of nude mice, and the tumor volume was measured every 3 days for a total of 30 days. The lower panel graph shows representative images and tumor volumes measured in xenograft nude mice at the specified time points. $N=5$. $(H)$ Top, images of the primary tumors of the footpad, metastatic tumors of the ankle, and distal lymph nodes that were stripped; bottom, average tumor weights measured after the end of the experiment $(N=5)$. ${ }^{*}, P<0.05$; $* *, P$ $<0.01 ; * \star *$ or $* \star \star \star, P<0.001 ;$ NS, not significant. 
A $\quad$ D4

D9

LOXL2

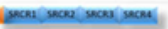

D4-HA D9-HA LOXL2-HA KDa

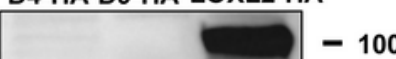

-100
-75

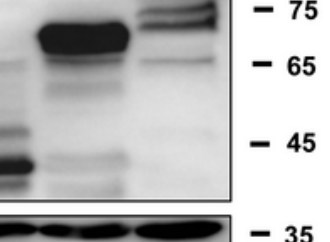

C

IB: HA $\mid$\begin{tabular}{ll|} 
LOXL2-HA & - \\
D9-HA &
\end{tabular}
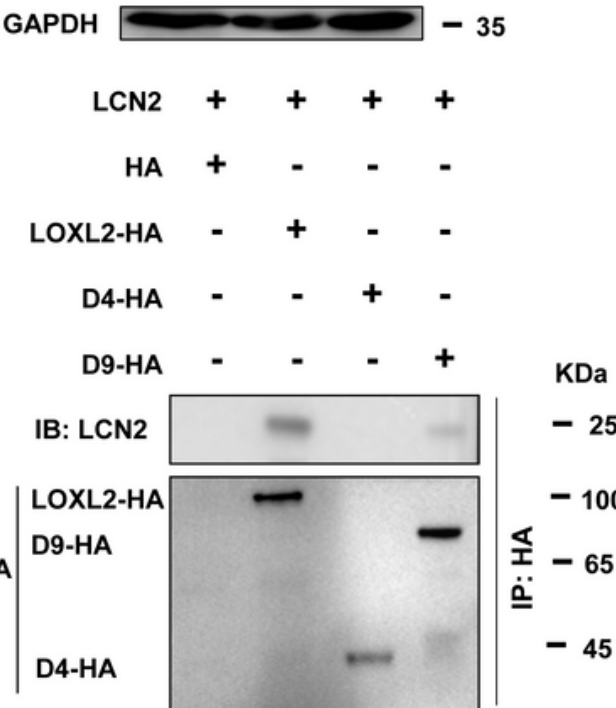

IB: HA $\mid \begin{aligned} & \text { LOXL2-HA } \\ & \text { D9-HA } \\ & \text { D4-HA }\end{aligned}$

IB: LCN2

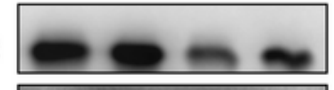

$-25$

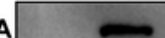

$-$

志- 100

E

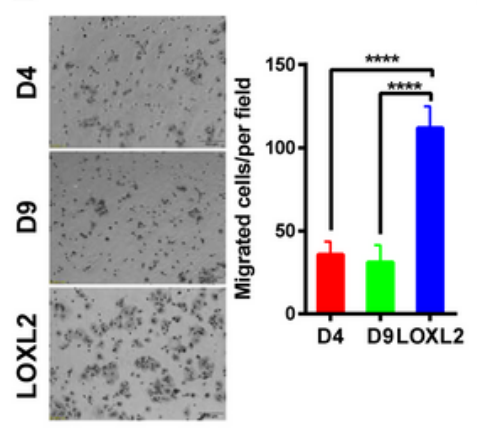

F

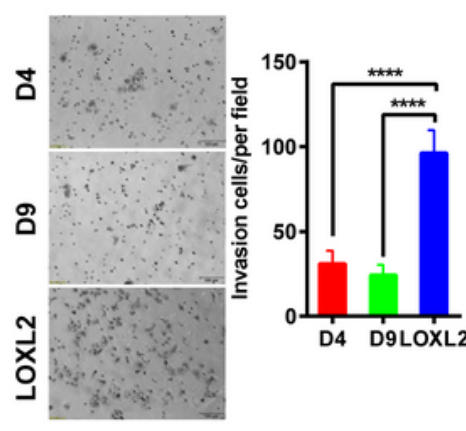

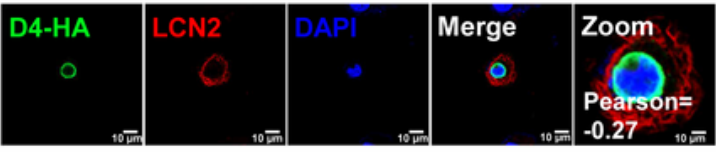

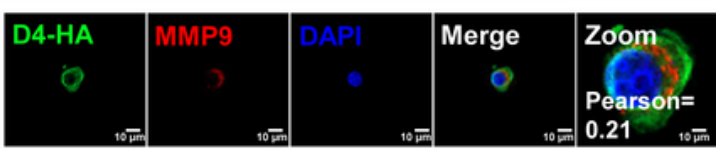

D
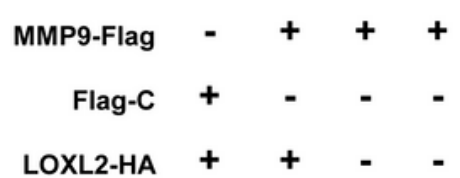

IB: HA D9-HA

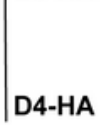

IB: Flag MMP9-Flag

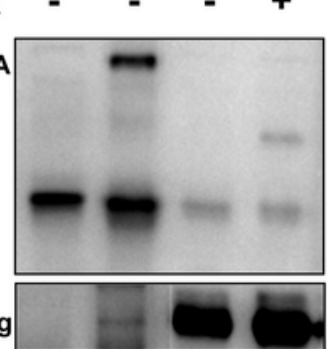

$\mathrm{KDa}$

$-100$

$\frac{\pi}{4}^{-65}$

$\cong-45$

$-100$

$-100$

䓂- 65

$-45$

$-100$

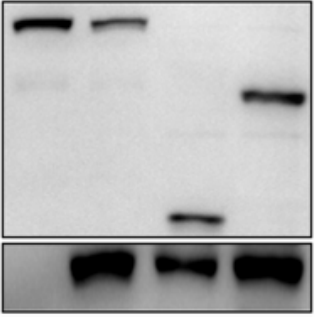

IB: Flag MMP9-Flag

G

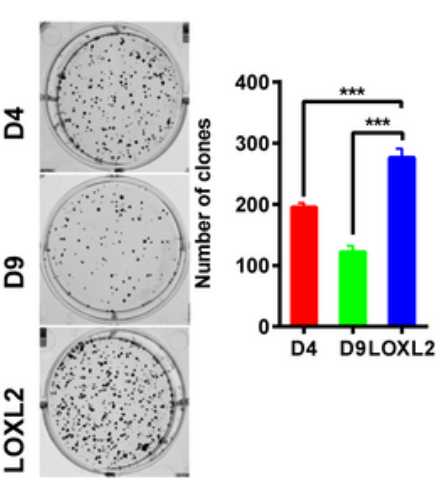

Figure 3

Identification of LOXL2/LCN2 and LOXL2/MMP9 protein-protein interaction domains. (A) Schematic diagram of truncated LOXL2 plasmid construction and their expression as identified by western blot. In the KYSE150 cell line, D4-HA/LCN2 or D4-HA/MMP9 were co-transfected, and their co-localization was observed by immunofluorescence. (C) LCN2/HA-V, LCN2/LOXL2-HA, LCN2/D4-HA, or LCN2/D9-HA were co-transfected. Co-IP was used to identify the domain necessary for LCN2 and LOXL2 protein-protein 
interaction. (D) LOXL2-HA/Flag-C, LOXL2-HA/MMP9-Flag, MMP9-Flag/D4-HA, or MMP9-Flag/D9-HA were co-transfected to identify the interaction domain between MMP9 and LOXL2. Migration (E) and invasion $(F)$ assays were used to compare the effects of truncated LOXL2 on the migration and invasion of cancer cells. $(G)$ Colony-forming assay was used to compare the effects on the proliferation of carcinoma cells. Each experiment was repeated at least three times. *, $\mathrm{P}<0.05 ; * \star, \mathrm{P}<0.01 ; * \star *$ or $* * \star *, \mathrm{P}<0.001$.

A

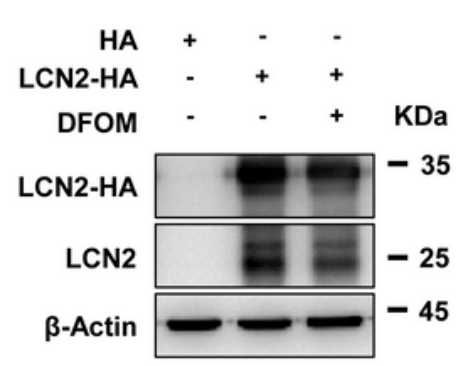

C

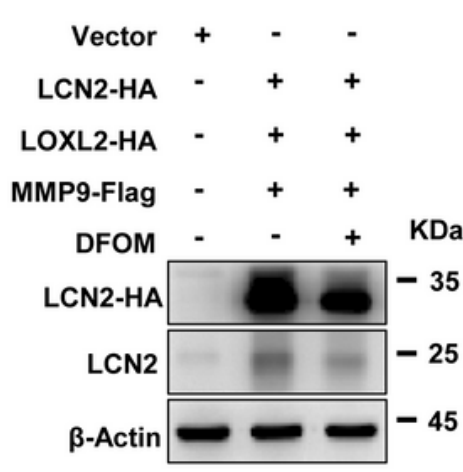

B
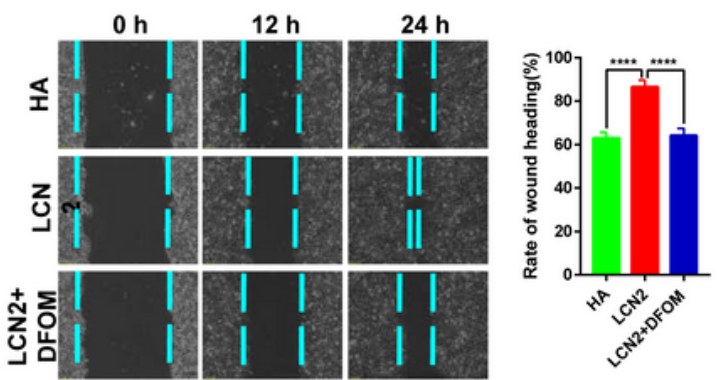

D

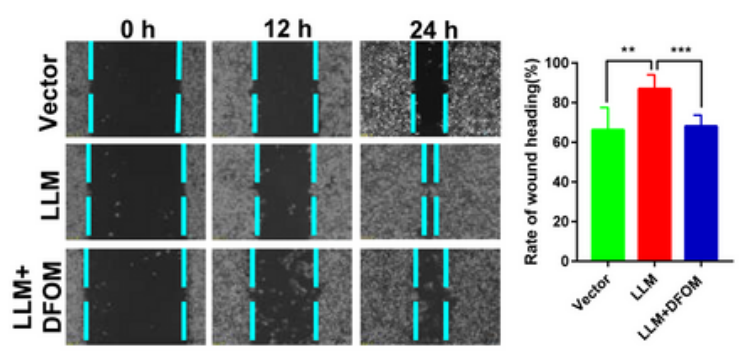

E
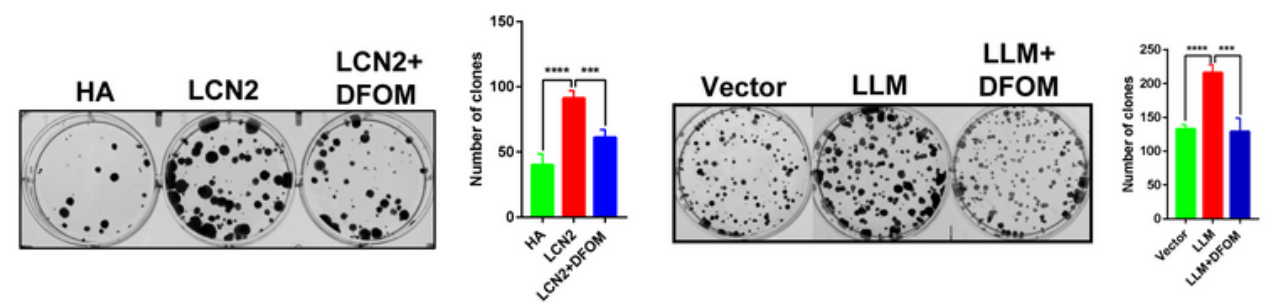

$\mathbf{F}$
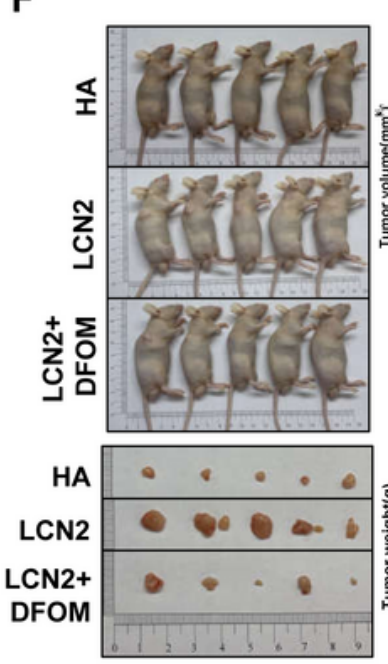

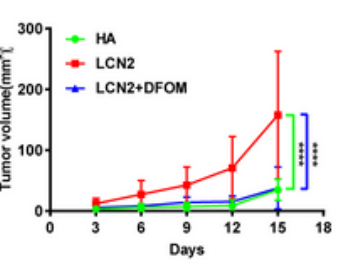

G

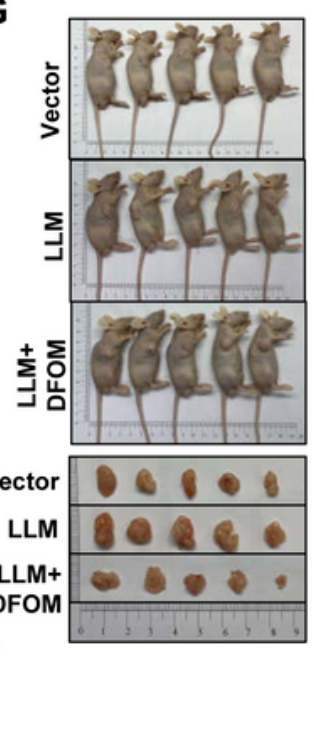

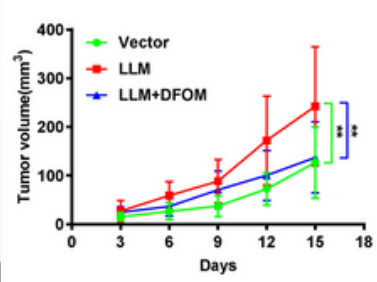

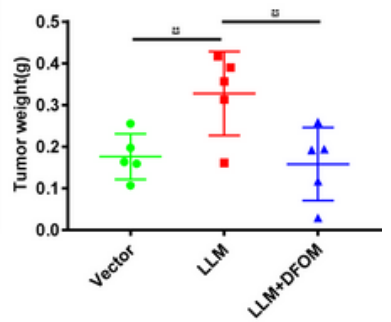

Figure 4 
DFOM inhibits the migration and tumor growth of esophageal cancer cells. In KYSE150 cells, LCN2 or LCN2/LOXL2/MMP9 was overexpressed and then cells were treated with DFOM (30 $\mu \mathrm{M})$ for 24 hours. Western blotting was used to detect the expression level of LCN2 (A, C), and a wound-healing assay was used to detect cell migration (B, D). (E) LCN2 or LCN2/LOXL2/MMP9 was overexpressed and treated with DFOM $(30 \mu \mathrm{M})$ for 3 days. Colony-formation assays were applied to detect cell proliferation. $100 \mu \mathrm{L}$ of KYSE150 (1× 107 cells/mL in serum-free medium) stably overexpressing LCN2 (F) or

LCN2/LOXL2/MMP9 (G) was injected into the right armpit of nude mice. The drug treatment began After three days. DFOM $(200 \mathrm{mg} / \mathrm{kg})$ or saline $(0.9 \% \mathrm{NaCl})$ was injected into the abdominal cavity for a total of 14 days, the nude mice were euthanized and tumors were stripped. Representative nude mouse and anatomical tumors are shown, and the nude mouse tumor volume at the specified time points and the

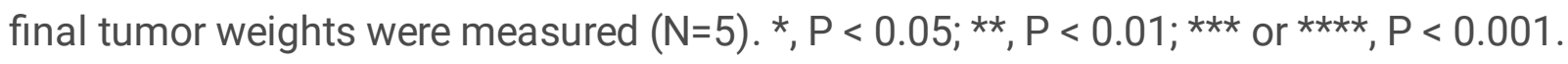



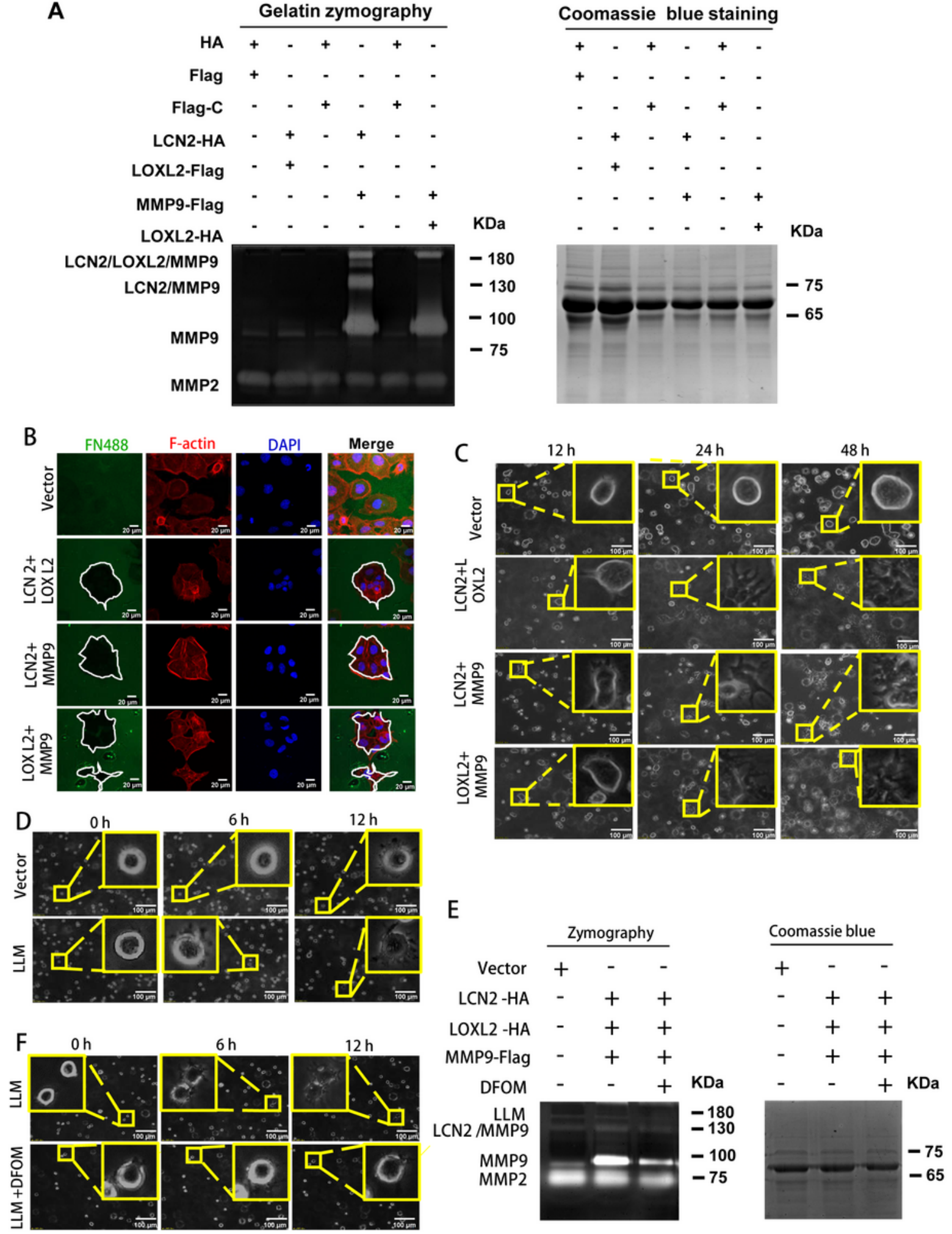

Figure 5

LCN2/LOXL2/MMP9 protein-protein interaction promotes extracellular matrix degradation. (A) Zymography (left panel) was applied to detect enzyme activities of MMP2 and MMP9, after the cooverexpression of LCN2/LOXL2, LCN2/MMP9, or LOXL2/MMP9, as indicated. The amount of loaded total protein is shown in the right panel. (B) Fluorescent matrix degradation was performed to observe ECM degradation. The white curvilinear frame indicates the matrix degradation area. FN488 Fluorescent matrix 
gel is shown in green, F-actin is stained in red, and nuclei are stained blue with DAPI. Scale, $20 \mu \mathrm{m}$. (C-D) LCN2/LOXL2, LCN2/MMP9, LOXL2/MMP9, LCN2/LOXL2/MMP9 were transfected as indicated. Overexpressing cells were mixed with Matrigel to construct a 3D culture model. Degradation of matrix and formation of cell filopodia were observed at different time points. (E) LCN2/LOXL2/MMP9 was overexpressed, and then cells were treated with DFOM $(30 \mu \mathrm{M})$ for $24 \mathrm{~h}$. The supernatant was collected and the enzymatic activity of MMP2 and MMP9 was detected by zymography. (F) Cells overexpressing LCN2/LOXL2/MMP9 were mixed with Matrigel to construct a 3D culture model, to observe the effect of DFOM on the degradation of the matrix and the formation of filopodia. 
A
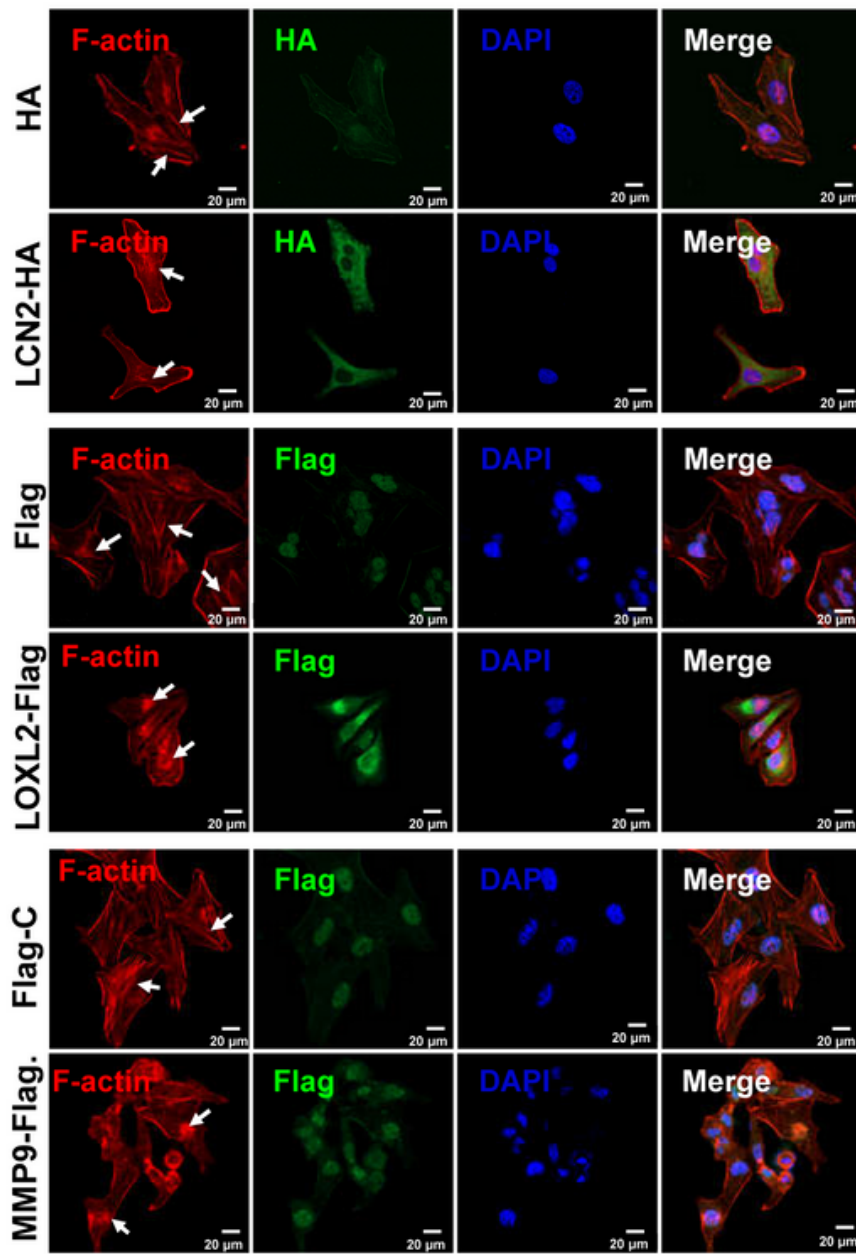

D
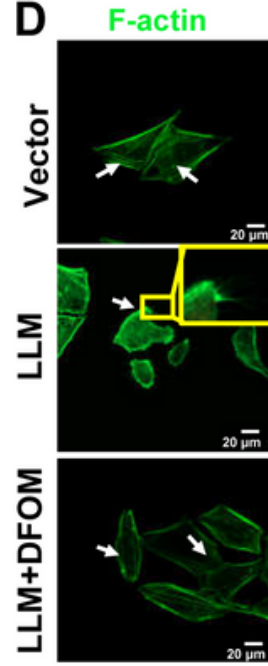

B
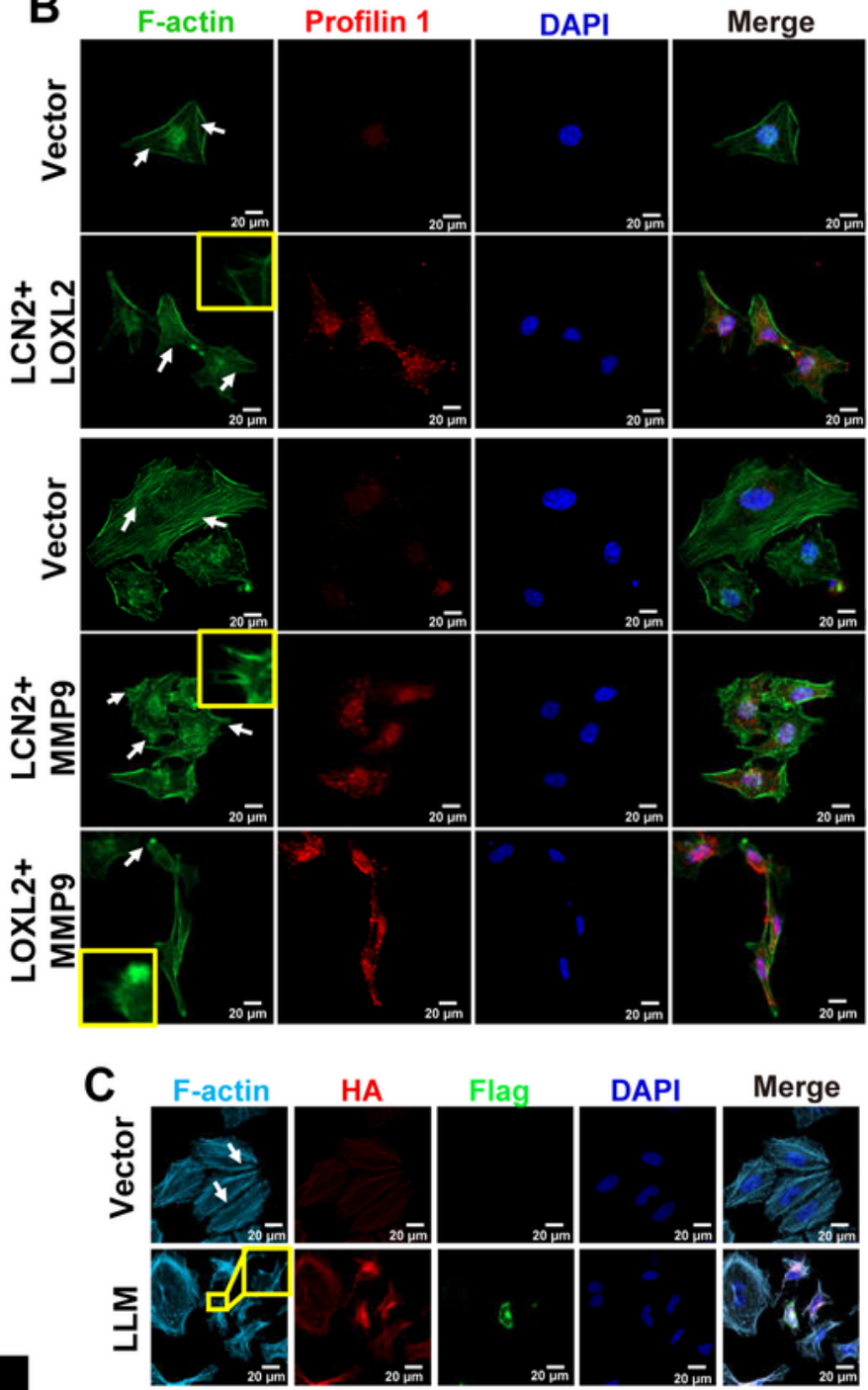

$\mathbf{E}$

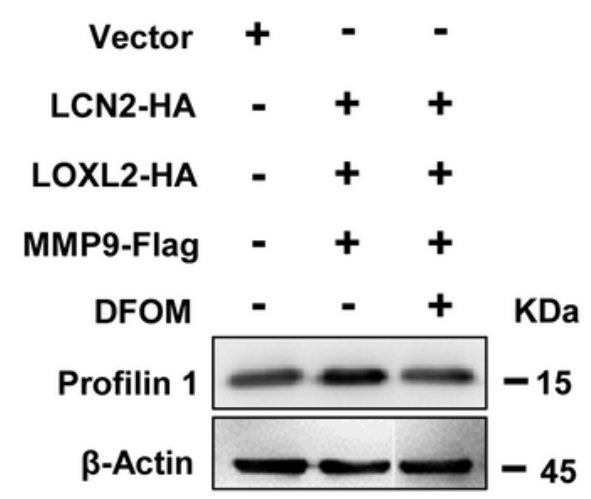

Figure 6

LCN2/LOXL2/MMP9 protein-protein interaction promotes microfilament remodeling. F-actin and profilin 1 were immunofluorescently labeled to detect their expression and distribution in cancer cells after transfection of LCN2, LOXL2 or MMP9, individually (A) and in combination (B), to analyze the rearrangement of the actin cytoskeleton. (C) LCN2-HA/LOXL2-HA/MMP9-Flag was overexpressed in KYSE410 cells, HA and Flag were fluorescently labeled using immunocytochemistry to observe the 
expression of the ternary complex, and F-actin was fluorescently labeled to observe the changes of the cytoskeleton. (D) Cells overexpressing LLM and treated with DFOM for 24 hours, then changes in F-actin and the expression and distribution of profilin 1 were observed. The white arrow points to the orderly microfilament skeleton in the cell, the yellow arrow indicates the disordered cytoskeleton or the elongated filopodia, and the yellow frame represents the partially enlarged view. Scale bar, $20 \mu \mathrm{m}$. (E) Western blot was used to detect the effect of DFOM on the expression of profilin 1 after LCN2-HA/LOXL2-HA/MMP9Flag overexpression.

A

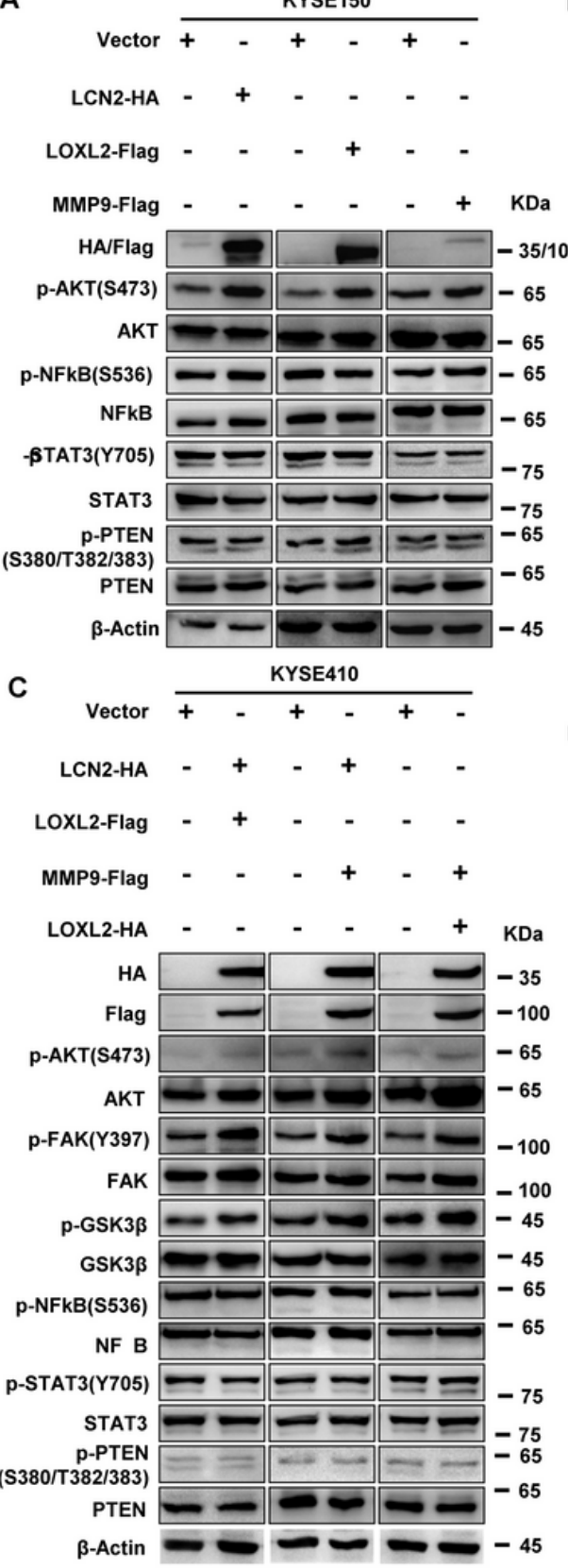

B
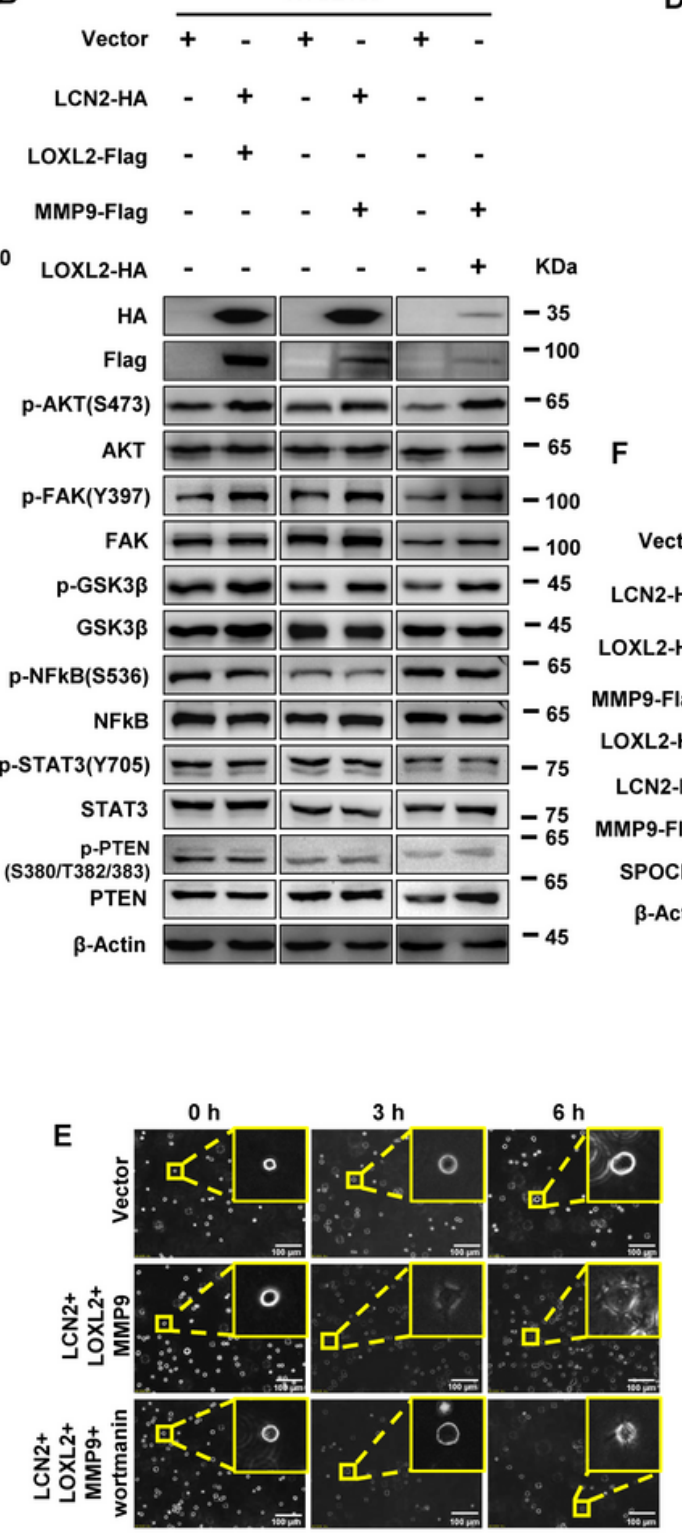

D $\frac{\text { KYSE150 }}{-\quad+}$ KDa

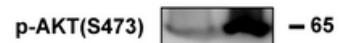

AKT -65

LCN2 -25

LOXL2 $\longrightarrow-75$

MMP9 -100

GAPDH -35

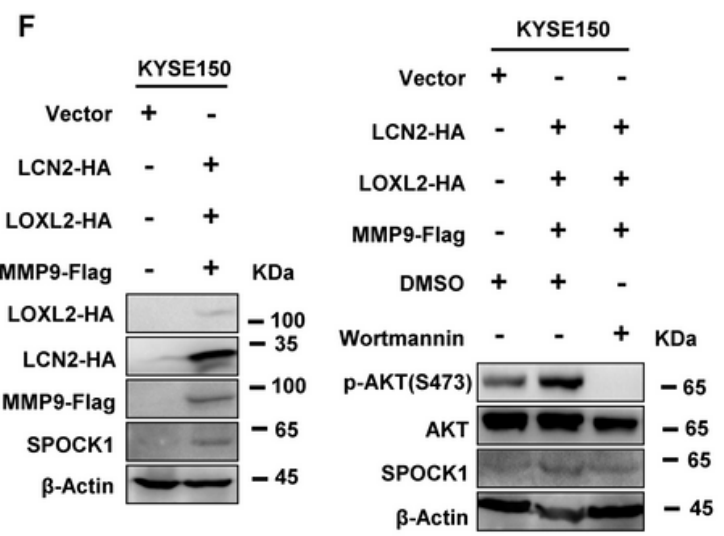

Figure 7

LCN2/LOXL2/MMP9 protein-protein interaction activates the FAK/AKT/GSK3 $\beta$ signaling pathway. (A) After overexpressing LCN2-HA, LOXL2-Flag, MMP9-Flag, the phosphorylation levels of AKT, NFkB, STAT3, and PTEN were detected. In KYSE150 (B) and KYSE410 (C) cells, the phosphorylation levels of AKT, FAK, 
GSK3 $\beta$, NFkB, STAT3, and PTEN were analyzed after the co-transfection of LCN2-HA/LOXL2-Flag, LCN2HA/MMP9-Flag, or LOXL2-HA/MMP9-Flag, respectively. (D) The pan-AKT kinase inhibitor capivasertib was added to KYSE150 cell to detect the expression of p-AKT (473), LCN2, LOXL2 and MMP9. (E) LCN2/LOXL2 /MMP9 were overexpressed in KYSE150 cells, and the treated group was treated with the PI3K inhibitor wortmannin. These cells were mixed with Matrigel to construct a 3D culture model, and cell morphological changes and the formation of filopodia were observed at different time points. $(F)$ Alteration of SPOCK 1 expression was detected after overexpressing LCN2-HA/LOXL2-HA/MMP9-Flag. Wortmannin, was added to detect the changes of phosphorylation levels of AKT, and the level of SPOCK1 as well. (G) Alteration of phosphorylated of AKT (473) was detected after overexpression of SPOCK1.

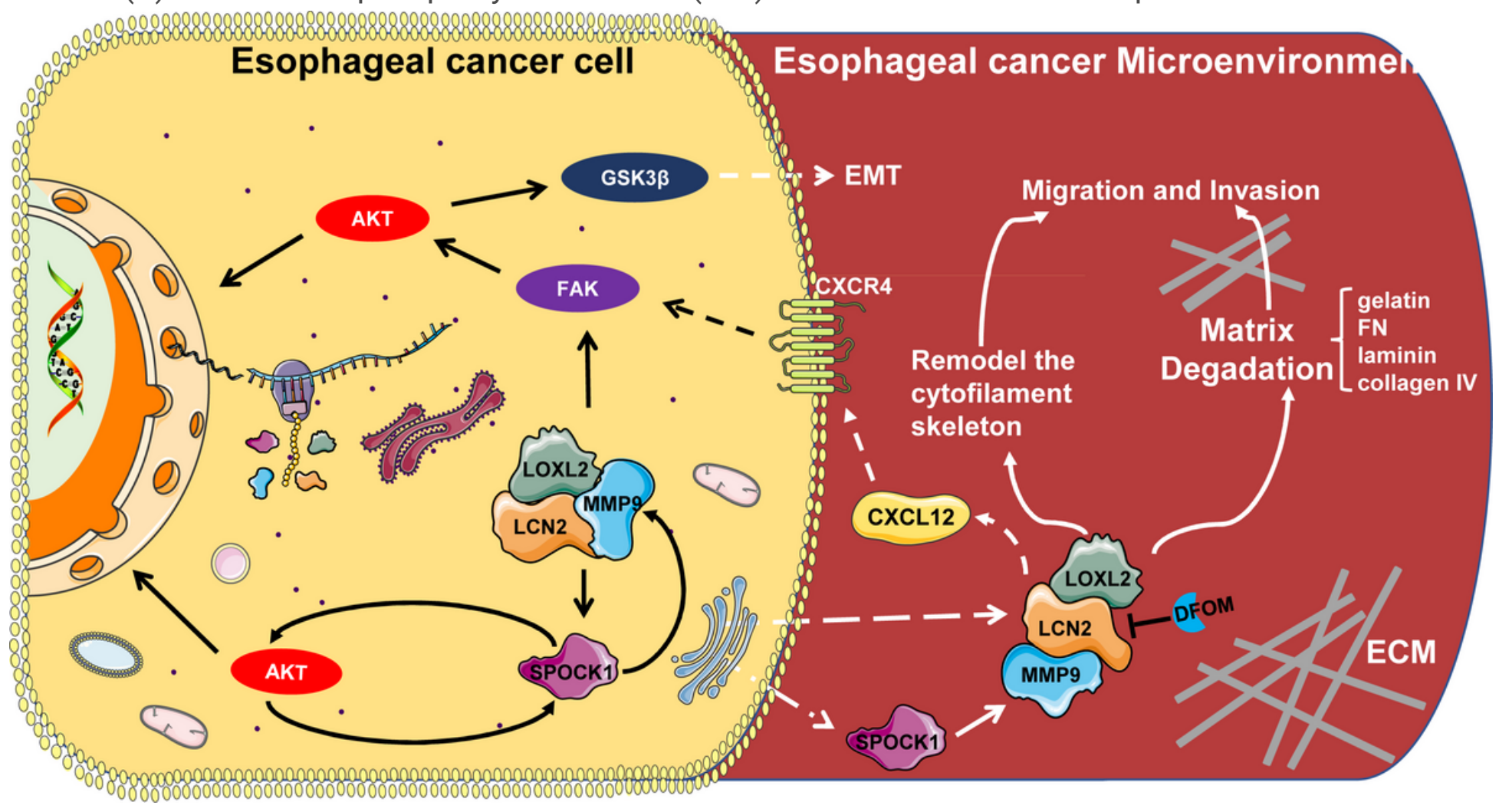

\section{Figure 8}

A model for LCN2/LOXL2/MMP9 complex enhancement of migration and invasion through the FAK/AKT/GSK3 $\beta$ signaling pathway and extracellular matrix degradation.

\section{Supplementary Files}

This is a list of supplementary files associated with this preprint. Click to download.

- SupplementaryFigureS1.tif

- SupplementaryFigureS2.tif

- SupplementaryFigureS3.tif

- SupplementaryFigureS4.tif 
- SupplementaryFigureS5.tif

- SupplementaryFigureS6.tif

- SupplementaryFigurelegends.docx

- SupplementaryTableS1.docx

- SupplementaryTableS2.docx 